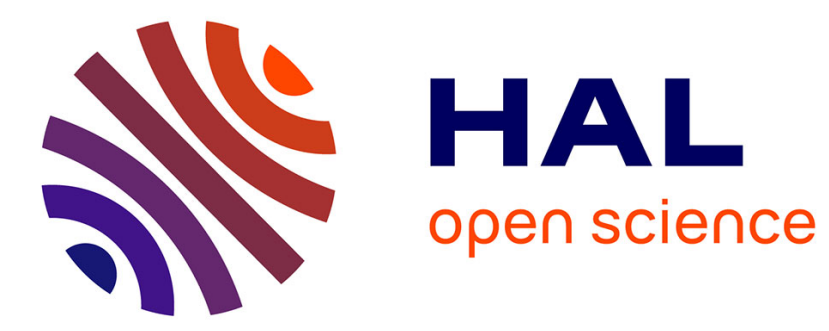

\title{
Multibody and macroscopic impact laws: a Convex Analysis Standpoint
}

Félicien Bourdin, Bertrand Antti Maury

\section{To cite this version:}

Félicien Bourdin, Bertrand Antti Maury. Multibody and macroscopic impact laws: a Convex Analysis Standpoint. Trails in Kinetic Theory, In press. hal-02555465v2

\section{HAL Id: hal-02555465 \\ https://hal.science/hal-02555465v2}

Submitted on 15 Jun 2020

HAL is a multi-disciplinary open access archive for the deposit and dissemination of scientific research documents, whether they are published or not. The documents may come from teaching and research institutions in France or abroad, or from public or private research centers.
L'archive ouverte pluridisciplinaire HAL, est destinée au dépôt et à la diffusion de documents scientifiques de niveau recherche, publiés ou non, émanant des établissements d'enseignement et de recherche français ou étrangers, des laboratoires publics ou privés. 


\title{
Multibody and macroscopic impact laws: a Convex Analysis Standpoint
}

\author{
Félicien Bourdin and Bertrand Maury
}

\begin{abstract}
These lecture notes address mathematical issues related to the modeling of impact laws for systems of rigid spheres and their macroscopic counterpart. We analyze the so-called Moreau's approach to define multibody impact laws at the mircroscopic level, and we analyze the formal macroscopic extensions of these laws, where the non-overlapping constraint is replaced by a barrier-type constraint on the local density. We detail the formal analogies between the two settings, and also their deep discrepancies, detailing how the macroscopic impact laws, natural ingredient in the so-called Pressureless Euler Equations with a Maximal Density Constraint, are in some way irrelevant to describe the global motion of a collection of inertial hard spheres. We propose some preliminary steps in the direction of designing macroscopic impact models more respectful of the underlying microscopic structure, in particular we establish micro-macro convergence results under strong assumptions on the microscopic structure.
\end{abstract}

\section{Introduction}

The modeling of particle systems spreads over a wide range of approaches, which rely on various levels of description of the particles. At one end of this range, the microscopic / Lagrangian setting is based on an individual description of particles, which "simply" obey Newton's Laws. At the other end, macroscopic models rely on a description of the collection of particles by a local density, and designing models amounts to elaborating equations verified by the velocity fields, under the implicit assumption that such a velocity is indeed well-defined. Between those extreme levels

F. Bourdin

Laboratoire de Mathématiques d'Orsay, Université Paris-Sud, e-mail: felicien.bourdin@ens.fr

B. Maury

Département de Mathématiques et Applications, Ecole Normale Supérieure, PSL University, 45 rue d'Ulm, 75005 Paris, e-mail: bertrand.maury@ens.psl.eu 
of descriptions, Boltzmann-like models are based on a kinetic description of the particle collection, namely a function $f(x, v, t)$ which quantifies at time $t$ the number of particles around $x$ at velocity $v$. Note that this setting makes it possible to handle a diffuse limit (smooth $f$ representing an infinite number of infinitely small particles), as well as finite collection (a single particle at a given velocity is represented as a Dirac mass in the $(x, v)$ space). From this standpoint, the kinetic description can be considered as microscopic in a generalized sense. This setting is particularly relevant to describe the limit of a low-density gas with the underlying hypothesis of elastic binary collisions between particles, and it is a natural bridge between Lagrangian models, considered as untractable for many-body systems, and macroscopic models which can be used to investigate the global behavior of these systems, by means of theoretical analysis or numerical computations. Considerable energy has been, and is still, deployed to rigorously obtain macroscopic models from the Boltzmann equation, like Euler or Navier-Stokes equations (see e.g. [15, 36]).

We are interested here in dense collections of finite size particles (more commonly called grains in this context), subject to possibly non-elastic collisions. The nondilute character of the collections together with the non-elastic character of the collision is likely to rule out the hypothesis of sole binary collisions which prevails in the Boltzmann context: multiple (or quasi-simultaneous) collisions together with persistent contacts can be expected to be generic in this situation. As a consequence, Boltzmann-like equations can no longer be considered as a natural step between microscopic and macroscopic models, and most macroscopic models which have been proposed to describe the behavior of dense (up to jammed) granular media have indeed been built independently from any homogenization procedure. We propose here to investigate the possibility to identify some ingredients that might appear in relevant macroscopic models considered as limits of microscopic ones. Let us make it clear that we are far from proposing a full and rigorous construction of a macroscopic model from the microscopic one, which is clearly out of reach. We shall rather focus on a crucial part of microscopic models, namely collision laws, and investigate the possibility to infer collision laws at the macroscopic level which would be respectful of the microscopic structure.

If one restricts to local interactions due to direct contact between entities, microscopic models based on finite size grains essentially rely on impact laws. Different strategies have been carried out to formalize this type of direct interactions. In the Molecular Dynamic approach (MD), see e.g. [1], one considers that grains are slightly deformable by implementing a short range force of the repulsive type. Note that this force is commonly taken as a computational trick to handle the non-overlapping constraint. This makes it possible to circumvent the very question of collisions, for it leads to classical Ordinary Differential Equations which fit in a classical theoretical framework (Cauchy-Lipschitz theory), and which can be solved by standard numerical schemes to perform actual computations. Note that, in spite of the natively elastic character of such interactions, some ingredients can be included to account for inelastic collision, as well as shear forces (see [29]).

In order to directly address micro-macro issues, we shall restrict ourselves here to alternative approaches, called Contact Dynamics (CD), based on a hard-sphere 
setting. As detailed in a recent review ([27]), several strategies can still be carried out to formalize the behavior of the system whenever the non-overlapping constraint is up to be violated. A popular, sometimes called event-driven, strategy, consists in handling binary collisions only. In this setting multiple collisions are considered as so rare that they can be disregarded, which makes it possible to use explicit expressions of post-collisional velocities. Another strategy is based on extending the so-called Darboux-Keller shock dynamics to multibody collisions. It consists in changing the time scale in the neighborhood of a collision event, to set it at the impulse scale. The dynamics is then described as a sequence of compression and extension phases (see [17] for a detailed description of this method). We also refer to [14] for a very detailed account of thermodynamical aspects of collision problems.

The developments we present here are based on an alternative approach, called Moreau's approach in [27], which considers instantaneous impacts involving an arbitrary large number of grains, treated in a global way (see [30, 26, 3]). As detailed below, it relies on basic concepts of Convex Analysis, the principal of which being the cone of feasible direction associated to the set of admissible configurations (configurations with no overlapping), and the associated polar cone (set of vector which have a nonpositive scalar product with all feasible directions) which is the outward normal cone. Given a restitution coefficient $e \in[0,1]$, the post-collisional velocity is determined from the projection of the pre-collisional velocity on the outward normal cone. Since everything can be written as a simple expression of the projection on the cone of feasible directions, we shall actually focus on this very notion in the largest part of these notes.

We shall end this introductory section by a few considerations on microscopic impact law following Moreau's approach, and what appears to be the canonical extension of this approach to the macroscopic setting. Section 2 is then dedicated to a detailed analysis on these impact laws. Identifying similarities and discrepancies between these formally similar laws is the object of Section 3. We describe in particular Laplace-like operators which are canonically associated to the collision laws in both settings, We introduce a notion of Abstract Maximum Principle (detailed in the appendix), which is verified in the macroscopic setting but not in the microscopic one, which deeply differentiates both models, and enlighten in some way the poorness of the macroscopic law.

In Section 4, we investigate the possibility to elaborate macroscopic impact models which are more respectful of the underlying microscopic structure. As detailed in Section 5, a rigorous homogenization procedure makes it possible to build such macroscopic models under very strong assumptions on the structure.

Although the resulting evolution problems are out of the scope of our work, we dedicate Section 6 to some remarks on this aspect of the problem. In the microscopic setting, the question is delicate but well understood: the problem is well-posed for analytic data, but might admit multiple solution otherwise, even for infinitely smooth data. In the macroscopic setting, under oversimplifying assumptions, the expected model takes the form of the so called pressureless equations with maximal density constraint, which essentially fits into a sound framework in the one-dimensional 
setting only $([7,4])$. For higher dimension, little is known on this equation. Let us add that the system is commonly written without any collision law, the actual choice being usually made in an implicit way, depending on the approach which is followed. For instance, in [7], particular solutions are built by means of sticky blocks with a purely inelastic collision law, whereas in $[10,11]$, the approach is based on compressible Euler equation with a barrier-like pressure with respect to the density, natively leading to a purely elastic behavior.

The largest part of this text is meant to be accessible to graduate students, so we tried to preserve self-consistency as far as possible, writing at some points full proofs of elementary results, in particular in the appendix.

\section{From single collision to multibody impact laws}

We introduce here Moreau's approach of impact laws, which fits in the general class of Contact Dynamics Methods (see [26, 31]). Let us start with a point particle subject to remain in the upper half plane $\mathbb{R} \times \mathbb{R}_{+}$, with a purely inelastic collision law on the boundary. We denote by $r=(x, y)$ its position, and by $u$ its velocity. If this particle is not subject to any force, its motion follows

$$
u^{+}=P_{C_{r}} u^{-}, \text {with } C_{r}=\mid \begin{array}{ll}
\mathbb{R}^{2} & \text { if } y>0 \\
\mathbb{R} \times \mathbb{R}_{+} & \text {if } y=0
\end{array}
$$

where $u^{-}$(resp. $u^{+}$) is the pre- (resp. post-) collisional velocity, and $P_{C_{r}}$ is the euclidian projection on $C_{r}$. When the particle does not touch the wall, the velocity is constant. When a collision occurs, with pre-collisional velocity $u^{-}=\left(u_{x}, u_{y}\right)$ (with $\left.u_{y}<0\right)$, the post-collisional velocity is $u^{+}=\left(u_{x}, 0\right)$.

In the case of an elastic collision, we introduce a restitution coefficient $e \in(0,1]$. The post-collisional velocity is now $u^{+}=\left(u_{x},-e u_{y}\right)$. This behavior can be written in a way which can generalized to the multi-collisional situation. We introduce the outward normal cone to $K$, defined as

$$
N_{r}=C_{r}^{\circ}=\left\{v \in \mathbb{R}^{2}, v \cdot w \leq 0 \quad \forall w \in C_{r}\right\}=\{0\} \times \mathbb{R}_{-} .
$$

The collision law can be written

$$
u^{+}=u^{-}-(1+e) P_{N_{r}} u^{-} .
$$

In the multi-collisional situation, the Moreau's approach consists in straightforwardly write the previous collision law, with the appropriate notion of cone of feasible velocities and outward normal cone. Consider a many-body system of hard spheres in $\mathbb{R}^{d}$, centered at $r_{1}, \ldots, r_{n}$, with common radius $R$. The feasible set writes

$$
K=\left\{r \in \mathbb{R}^{d n}, D_{i j}=\left|r_{j}-r_{i}\right|-2 R \geq 0 \quad \forall i \neq j\right\} .
$$


Fig. 1 Collision against a wall. Depending on the restitution coefficient $e \in[0,1]$, the post collisional velocity can take any value between $\left(u_{x}, 0\right)$ (purely non-elastic) and $\left(u_{x},-u_{y}\right)$ (elastic).

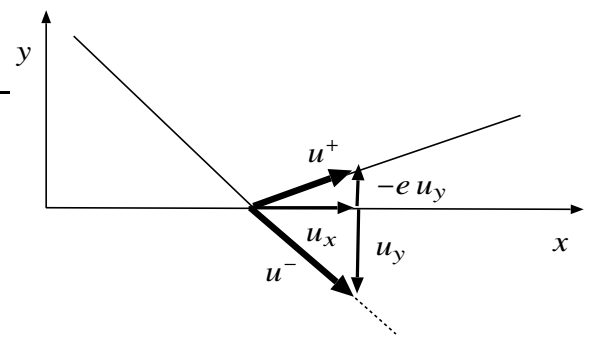

Denoting $e_{i j}=\frac{r_{j}-r_{i}}{\left|r_{j}-r_{i}\right|}$, the set of admissible velocities is

$$
C_{r}=\left\{v, D_{i j}(r)=\left|r_{j}-r_{i}\right|-2 R=0 \Rightarrow e_{i j} \cdot\left(v_{j}-v_{i}\right) \geq 0\right\} .
$$

(N.B.: we use the notation $a \cdot b$ to denote the dot product of vectors in the physical space $\mathbb{R}^{d}$, while $\langle\cdot \mid \cdot\rangle$ shall be used for generalized velocity vectors in $\mathbb{R}^{n d}$, or elements in abstract Hilbert spaces.)

Let $r=\left(r_{1}, \ldots, r_{n}\right) \in K$ be given. As previously, the outward normal cone to $K$ at $r$ is defined as the polar set to the cone of feasible velocities:

$$
N_{r}=C_{r}^{\circ}=\left\{v \in \mathbb{R}^{d n},\langle v \mid w\rangle \leq 0 \quad \forall w \in C_{r}\right\} .
$$

To alleviate notation, we shall now denote by $U$ the pre-collisional velocity, and by $u$ the post-collisional velocity. With these new notation, the collision model writes

$$
u=U-(1+e) P_{N_{r}} U,
$$

where $e \in[0,1]$ is the restitution coefficient. Since $N_{r}$ and $C_{r}$ are mutually polar, it holds that

$$
I=P_{N_{r}}+P_{C_{r}},
$$

where $I$ is the identity operator in $\mathbb{R}^{d n}$ (see [25], or the proof of Proposition 11 in the appendix). As a consequence, the post-collisional velocity can be expressed in terms of $P_{C_{r}} U$, for any $e \in[0,1]$,

$$
u=U-(1+e)\left(U-P_{C_{r}} U\right),
$$

which simply reduces to $u=P_{C_{r}} U$ for $e=0$. For the sake of simplicity, we shall therefore focus on this purely inelastic situation, keeping in mind that the knowledge of $P_{C_{r}} U$ makes it possible to recover the whole range of elastic collision laws through (4). 


\section{Macroscopic impact laws}

We describe here informally how the Moreau's approach described above can be developed at the macroscopic scale. More details will be given in the next section. We consider an infinite collection of inertial grains described by a macroscopic density $\rho$, which is subject to remain below a prescribed value, which we set at 1 . We denote by $\widehat{K}$ the corresponding set of densities of a given mass, which are assumed to be supported in some domain $\Omega$.

We denote by $U$ the velocity field at some instant, defined on the support of $\rho$, and we aim at defining a collision law which would give us the post-collisional velocity from this pre-collisional velocity $U$. In the purely inelastic setting, a natural candidate for this law amounts to define the post-collisional velocity $u_{+}$as the projection $u$ of $U$ on the set of all those vector fields which have a nonnegative divergence on the saturated zone, that is the macroscopic counterpart of $C_{r}$ (defined by (2)). Indeed, having $\nabla \cdot u<0$ in the neighborhood of some point in the saturated zone would lead to an increase of $\rho$, thereby a violation of the constraint. As will be detailed below, this cone $\widehat{C}_{\rho}$ can be described as the set of all those velocity fields which have a nonnegative divergence (in a weak sense) over the saturated zone.

\section{A closer look to micro and macro impact laws}

In this Section, we give some details on the mathematical formulation of the impact laws presented above, in the microscopic and macroscopic settings, and we investigate their similarities and discrepancies.

\subsection{Saddle point formulation of the microscopic impact law}

We consider as previously a system of hard spheres in $\mathbb{R}^{d}$, centered at $r_{1}, \ldots, r_{n}$, with common radius $R$. The feasible set writes

$$
K=\left\{r \in \mathbb{R}^{d n}, D_{i j}=\left|r_{j}-r_{i}\right|-2 R \geq 0 \quad \forall i \neq j\right\} .
$$

The set of feasible velocities $C_{r}$ is defined by (2). Let us denote by $m \in \mathbb{N}$ the number of contacts, i.e. the number of pairs $\{i, j\}$ such that $D_{i j}=\left|r_{j}-r_{i}\right|-2 R=0$. We introduce $B \in \mathcal{M}_{m, n}(\mathbb{R})$ the matrix which expresses the constraints, each row of which is

$$
G_{i j}=\left(0, \ldots, 0,-e_{i j}, 0, \ldots, 0, e_{i j}, 0, \ldots, 0\right) \in \mathbb{R}^{d n},
$$

where $e_{i j}=\left(r_{j}-r_{i}\right) /\left|r_{j}-r_{i}\right|$. The feasible set can be written

$$
C_{r}=\{v, B v \leq 0\}=B^{-1} \Lambda_{+}^{o}, \Lambda_{+}=\mathbb{R}_{+}^{m},
$$


Fig. 2 In the configuration represented here, the number of primal degrees of freedom is $2 \times n=28$, whereas the number of contacts is $m=29$.

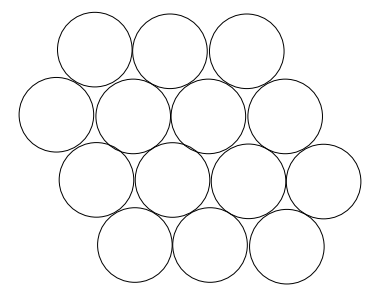

where $\Lambda_{+}^{\circ}$ is the polar cone to $\Lambda_{+}$, that is $\mathbb{R}_{-}^{m}$, and $B^{-1} \Lambda_{+}^{\circ}$ its preimage by $B$.

The problem which consists in projecting $U \in \mathbb{R}^{\text {nd }}$ on $C_{r}$ fits into the abstract setting of Proposition 12 in the appendix, and it can be put in a saddle point form:

Proposition 1 Let $C_{r} \in \mathbb{R}^{\text {dn }}$ be defined by (2) (or equivalently by (7)). Denote by $B^{\star}$ the transpose of the matrix $B$. If $u=P_{C_{r}} U$ then there exists $p \in \Lambda_{+}=\mathbb{R}_{+}^{m}$ such that

$$
\mid \begin{aligned}
& u+B^{\star} p=U \\
& B u \leq 0 \\
& \langle B u \mid p\rangle=0 .
\end{aligned}
$$

Conversely, if $(u, p) \in \mathbb{R}^{d n} \times \mathbb{R}_{+}^{m}$ is a solution to (8), then $u=P_{C_{r}} U$.

Proof Let us start by a preliminary remark: the fact that the image of $B^{\star}$ is closed (it is a finite dimensional linear space) is not sufficient to ensure that $B^{\star}\left(\Lambda_{+}\right)$is closed (see Remark 11 in the appendix). This property is nevertheless true here, because $B^{\star}\left(\Lambda_{+}\right)$is spanned by a finite number of vectors

$$
B^{\star}\left(\Lambda_{+}\right)=\left\{-\sum_{i j} p_{i j} G_{i j}, p_{i j} \geq 0\right\}
$$

where $G_{i j}$ is defined by (6), which implies closedness by Lemma 3. Proposition 12 then ensures existence of $p \in \Lambda_{+}=\mathbb{R}_{+}^{m}$ such that $u+B^{\star} p=U$, with the complementarity condition $\langle B u \mid p\rangle=0$.

If we furthermore assume that $B^{\star}$ is one-to-one, i.e. $B$ is onto, then $p$ is unique. The one-to-one character of $B^{\star}$ is lost as soon as the number of constraints is larger than the number of degrees of freedom (hyperstatic situation). For identical disks in $2 \mathrm{~d}$, it can appear as soon as $n=14$ discs are involved (see Figure 2). For many-body triangular lattices, the number of primal degrees of freedom is $2 n$, while the number of constraints is asymptotically $3 n$, which mean that the dimension of the kernel of $B^{\star}$ is asymptotically $n$.

The problem nevertheless presents some sort of uniqueness property, restricted to the homogeneous problem $(U=0)$. The following proposition essentially states a very intuitive fact: if one considers any static configuration of a finite number of hard spheres in the open space (i.e. with no walls), under the assumption that interaction 
contact forces are only repulsive, then all forces are actually zero. This property will be used to show that the solution set for the pressure field (Proposition 1) is bounded.

Lemma 1 We consider an admissible configuration $r \in K$, and the associated matrix $B \in \mathcal{M}_{m, n}(\mathbb{R})$ (the raws of which are given by (6)). The set

$$
S=\left\{q \in \mathbb{R}_{+}^{m}, B^{\star} q=0\right\}=\operatorname{ker} B^{\star} \cap \Lambda_{+}
$$

is reduced to $\{0\}$.

Proof Let us first establish the uniqueness for the homogeneous problem. We consider $q=\left(q_{i j}\right) \in \mathbb{R}_{+}^{m}$ such that

$$
B^{\star} q=\sum_{i \sim j} q_{i j} G_{i j}=0 .
$$

where $i \sim j$ means that the particles $i$ and $j$ are in contact. Let $i_{0}$ denote the index of an extremal vertex of the convex hull $\operatorname{conv}\left(q_{i}, 1 \leq i \leq n\right)$. By Hahn-Banach's theorem, the compact $\left\{q_{i_{0}}\right\}$ and the set $\operatorname{conv}\left\{q_{i}, i \neq i_{0}\right\}$, which is closed and convex, can be separated in a strict sense by a plane in $\mathbb{R}^{d}$. We denote by $x$ an element of this plane, and by $v$ a normal vector to it. One has

$$
\left(q_{i_{0}}-x\right) \cdot v>0,\left(q_{j}-x\right) \cdot v<0 \quad \forall j=1, \ldots, n, j \neq i_{0},
$$

so that $\left(q_{i_{0}}-q_{j}\right) \cdot v>0$ for $j \neq i_{0}$. Now the balance of contact forces exerted upon sphere $i_{0}$ in the direction $v$ reads

$$
\sum_{j \neq i_{0}} q_{j i_{0}} e_{j i_{0}} \cdot v
$$

where $e_{j i_{0}} \cdot v>0$, and $q_{j i_{0}} \geq 0$ for all $j$. This quantity is positive unless $q_{j i_{0}}=0$ for all $j \neq i_{0}$. Therefore all multipliers associated to a contact with sphere $i_{0}$ are equal to 0 , and this approach can be iterated for the reduced family $\left(q_{j}, j \neq i_{0}\right)$. By downward induction on the number of active spheres, we prove that $S$ is reduced to $\{0\}$.

An important consequence of this expected property is the boundedness of the solution set for (8).

Proposition 2 Under the assumptions of Proposition 1, the solution set for the dual component $p$

$$
S=\left\{q \in \mathbb{R}_{+}^{m}, B^{\star} q=U-u\right\}=\left(p+\operatorname{ker} B^{\star}\right) \cap \Lambda_{+},
$$

where $(u, p)$ is a solution to (8), is bounded.

Proof This is a direct consequence of Proposition 15 (in the appendix) and Lemma 1. 


\subsection{Saddle point formulation of the macroscopic impact law}

In the macroscopic setting, we consider that the solid phase is represented by a density supported in a domain $\Omega \in \mathbb{R}^{d}$, subject to remain below the value 1 . We denote by $\widehat{K}$ the set of all those measures, which is the macroscopic counterpart of the set $K$ of $n$-sphere configurations with no overlapping. We shall disregard here issues possibly related to wall conditions or mass at infinity: we assume that $\Omega$ is bounded, and that that the support of $\rho$ is strongly included in $\Omega$ (i.e. the support of $\rho$ is at a positive distance from $\partial \Omega$, which we denote by $\omega \subset \subset \Omega$ ).

We define a pre-collisional configuration as a density in $\widehat{K}$, together with a precollisional velocity field

$$
U \in L_{\rho}^{2}(\Omega)^{d}=\left\{v: \Omega \rightarrow \mathbb{R}^{d}, \rho \text { - measurable, } \int_{\Omega}|v|^{2} d \rho<\infty\right\} .
$$

We describe here a natural way to define a post collisional velocity $u$, natural in the sense that it directly follows the same principles as the microscopic law. This strategy to define a post-collisional velocity in the purely inelastic setting follows the framework proposed in $[22,23]$ for macroscopic crowd motion models. Feasible velocities are those which are non-concentrating in the saturated zone $[\rho=1]$. For smooth velocities, it amounts to prescribe a nonnegative divergence in this zone. Such a set can be properly defined by duality as

$$
\widehat{C}_{\rho}=\left\{v \in L_{\rho}^{2}(\Omega)^{d}, \int_{\Omega} v \cdot \nabla q d \rho \leq 0 \quad \forall q \in \Lambda_{\rho}, q \geq 0 \quad \text { a.e. }\right\}
$$

where the space $\Lambda_{\rho}$ of pressure test functions is defined as

$$
\Lambda_{\rho}=\left\{p \in H^{1}(\Omega), p(1-\rho)=0 \text { a.e. }\right\} .
$$

Note that, since we assumed that the support of $\rho$ is strongly included in $\Omega$, it holds that $\Lambda_{\rho} \subset H_{0}^{1}(\Omega)$.

It can be easily checked that, for a smooth velocity field $v$ and a regular saturated zone, belonging to $\widehat{C}_{\rho}$ is equivalent to verifying $\nabla \cdot v \geq 0$ on $[\rho=1]$.

The non-elastic collision law writes

$$
u=P_{\widehat{C}_{\rho}} U
$$

where the projection $P_{\widehat{C}_{\rho}}$ is with respect to the $L_{\rho}^{2}$ norm.

Let us check that it fits into the abstract setting of Proposition 12. with $V=L_{\rho}^{2}(\Omega)^{d}$, and $\Lambda=\Lambda_{\rho}$. We define $\Lambda_{+} \subset \Lambda_{\rho}$ as the set of all those functions in $\Lambda_{\rho}$ which are nonnegative almost everywhere:

$$
\Lambda_{+}=\left\{q \in \Lambda_{\rho}, q(x) \geq 0 \text { a.e. in } \Omega\right\} \text {. }
$$

We introduce 


$$
\widehat{B}: v \in V=L_{\rho}^{2}(\omega)^{d} \longmapsto \widehat{B} v \in \Lambda^{\prime},
$$

where $\widehat{B} v$ is defined by

$$
\langle\widehat{B} v, p\rangle=\int v \cdot \nabla p d \rho
$$

Note that $\Lambda$ and $\Lambda^{\prime}$ are not identified here, and that $\widehat{B}$ maps $V$ onto $\Lambda^{\prime}$, so that the adjoint operator $\widehat{B}^{\star}$ is defined in $\mathcal{L}(\Lambda, V)$, the set of continuous linear mappings from $\Lambda$ into $V$.

The saddle-point formulation of the problem can be written

$$
\mid \begin{array}{rlr}
u+\nabla p & =U & \rho \text {-a.e. in } \Omega, \\
\cdots-\nabla \cdot u & \leq 0 & \text { in }[\rho=1] ”, \\
p & \geq 0 & \rho \text {-a.e. in } \Omega, \\
\int_{\Omega} u \cdot \nabla p d \rho & =0,
\end{array}
$$

where the second equation (between quotation marks) is meant in a weak sense, i.e.

$$
\int_{\Omega} u \cdot \nabla q d \rho \leq 0 \quad \forall q \in \Lambda_{+} .
$$

This condition can also be written in an abstract way: $\widehat{B} u \in \Lambda_{-}$, where $\Lambda_{-}$is the polar cone to $\Lambda_{+}$, i.e. the cone of all those linear forms in $\Lambda^{\prime}$ which are nonpositive over $\Lambda_{+}$.

We may now state the well-posedness result.

Proposition 3 Let $\rho \in \widehat{K}$ be given as a density defined over a bounded domain $\Omega$, with $\operatorname{supp}(\rho)$ strongly included in $\Omega$. Problem (14) admits a unique solution $(u, p) \in V \times \Lambda_{+}$, and the primal component $u$ of this solution is the projection of $U$ on $\widehat{C}_{\rho}$.

Proof From Proposition (12) (more precisely Corollary 1), it is sufficient to prove that $\widehat{B}$ (defined by (13)) is onto. Let us prove that there exists a constant $\beta>0$ such that for every $q \in \Lambda$

$$
\left\|\widehat{B}^{\star} q\right\|_{L_{\rho}^{2}} \geq \beta\|q\|_{H^{1}}
$$

which writes $\|\nabla q\|_{L_{\rho}^{2}} \geq \beta\|q\|_{H^{1}}$ in the present context. Due to Poincaré Inequality, which holds true because $\Lambda \subset H_{0}^{1}(\Omega)$, it is sufficient to establish that the inequality $\|\nabla q\|_{L_{\rho}^{2}} \geq \beta\|\nabla q\|_{L^{2}}$ holds for any $q \in \Lambda$.

For $q \in \Lambda$, by Theorem 1.56 in [35] one has $(1-\rho) \nabla q=(1-\rho) \mathbf{1}_{q \neq 0} \nabla q=0$, so that $\|\nabla q\|_{L_{\rho}^{2}}=\|\nabla q\|_{L^{2}}$. As a consequence, $\widehat{B}^{\star}$ has a closed range, and so does $\widehat{B}$ by Banach-Steinhaus Theorem. The range of $\widehat{B}$ is also dense thanks to the same inequality, thus $\widehat{B}$ is onto. 


\section{Micro-macro issues}

We detailed in the previous section impact laws for a collection of rigid spheres, in the Moreau's spirit, and we proposed a natural instantiation of the same principles at the macroscopic level. The macroscopic version may appear as a natural candidate to handle collision between clusters of infinitely many hard spheres represented by a diffuse density. We shall see here that some considerations may comfort this standpoint in the one-dimensional setting. Yet, for dimensions $d \geq 2$, we shall prove that the macroscopic law presented in the previous section is not a relevant model for describing the impact between large collections of hard spheres.

\section{One dimensional setting}

In the one-dimensional setting (hard spheres move on a fixed line) the two approaches are mutually consistent, as we shall see here.

First, the notion of maximal density is well defined at the microscopic level: a cluster of spheres (represented by segments in 1d) is saturated if the solid phase covers some zone of the real line, which corresponds to $\rho=1$ in the macroscopic setting.

Now consider such a cluster of $n$ segments covering an interval $I \in \mathbb{R}$, and the corresponding macroscopic density $\rho=\mathbf{1}_{I}$ (characteristic function of $I$ ). We consider a pre-collisional velocity field $U$ that pushes the configuration against the boundary of the feasible set, i.e. such that $\partial_{x} U \leq 0$. In this case the constraint will be saturated overall the cluster so that, at the macroscopic scale, $-\nabla \cdot u=-\partial_{x} u=0$, and Problem (14) is a classical Darcy problem

$$
\mid \begin{aligned}
u+\partial_{x} p & =U, \\
-\partial_{x} u & =0
\end{aligned} \quad \text { in } I .
$$

Eliminating the velocity yields a Poisson problem on the pressure

$$
-\partial_{x x} p=-\partial_{x} U
$$

with Dirichlet boundary conditions on the boundary of $I$.

At the microscopic level, we simply consider pre-collisional velocities $U_{1}, \ldots$, $U_{n}$, with $U_{i}=U\left(q_{i}\right)$, and we make a slight abuse of notation by keeping $U$ to denote the vector of velocities. Since the velocities $U_{1}, \ldots, U_{n}$, are non-increasing, the constraint will also be saturated, which leads to a Darcy-like problem

$$
\mid \begin{aligned}
u+B^{\star} p & =U \\
B u & =0
\end{aligned}
$$

Eliminating the velocity yields a Poisson-like problem 

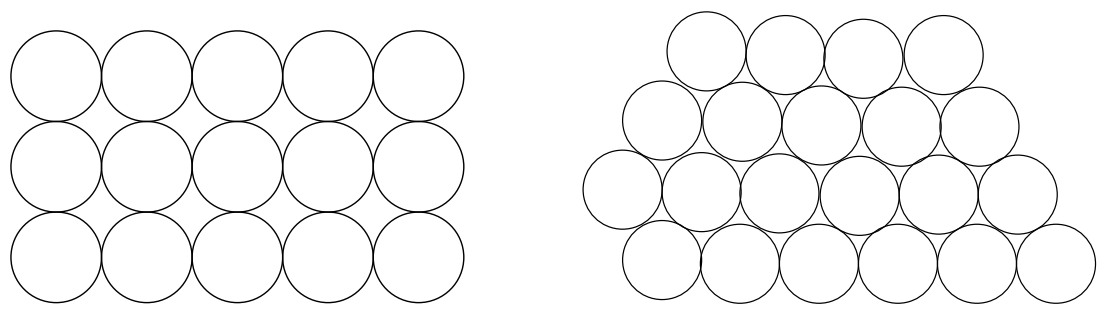

Fig. 3 Square (left) and triangular (right) lattices.

$$
B B^{\star} p=B U,
$$

with

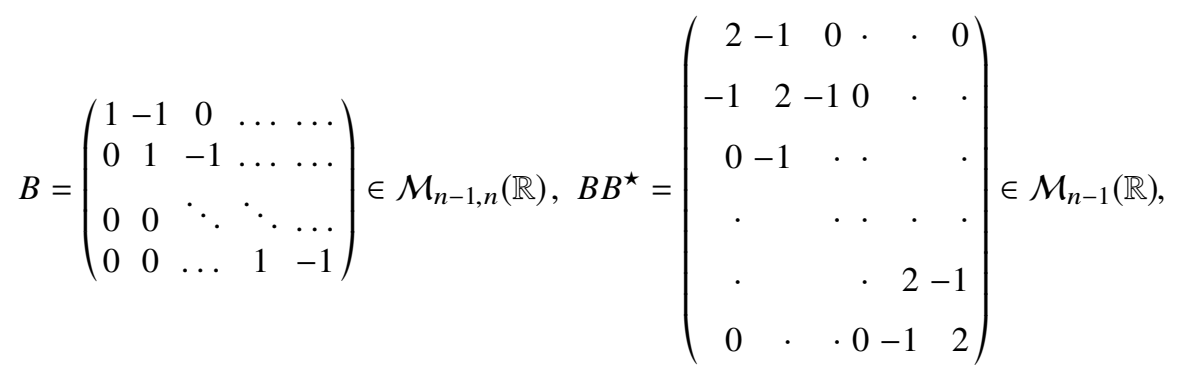

that is the discrete Laplacian matrix. The two formulations are mutually consistent in the sense that the linear system (18) is a standard finite difference discretization of the Poisson problem (16), which is covered by rigorous convergence results (see e.g. [2]).

\section{Case $d \geq 2$}

In higher dimensions the situation is fully different. First, the notion of maximal density is not clearly defined at the microscopic level. Let us consider collections of identical discs. The maximal packing density $\rho_{\max }=\pi / 2 \sqrt{3} \approx 0.9069 \ldots$, and corresponds to the triangular lattice (see Fig. 3, right). Yet the actual density of moving collections of rigid disks is generally strictly less than this maximal value, which does not mean that the flow is unconstrained (as the macroscopic setting would suggest). These considerations call for a clear identification of configurations which saturate the constraint. It is tempting to consider as maximal in some sense any density corresponding to such configurations, for which there are no free disks, so that constraints are activated everywhere. The triangular lattice is clearly jammed, but so is the cartesian lattice ( $\rho=\pi / 4 \approx 0.79$ ), and it is possible to build looser jammed configurations, for example by removing some non neighboring discs from 
the triangular lattice. We refer to [34] for a general review on the notion of maximal random packing.

Beyond this difficulty to properly define the notion of maximal density, the microscopic and macroscopic projections exhibit deep discrepancies in dimensions higher than 1 . We propose here to enlighten these discrepancies by considering the underlying Poisson problems for the pressure in both settings. Like in the one-dimensional setting, we first consider the macroscopic setting, which is in some manner simpler than the microscopic one, in spite of its infinite dimensional character.

The pressure can be shown, under some assumptions, to verify a Poisson like problem in the saturated zone, The first step consists in proving that the problem verifies the abstract maximum principle (see Definition 3), that is

$$
\widehat{B} U \in-\Lambda_{-}=-\Lambda_{+}^{\circ} \Longrightarrow \exists p \in \Lambda_{+} \text {s.t. } \widehat{B} \widehat{B}^{\star} p=\widehat{B} U .
$$

Proposition 4 We assume that $\operatorname{supp}(\rho)$ is strongly included in $\Omega$, and that $\Omega$ is connected. The couple $\left(\widehat{B}, \Lambda_{+}\right)$verifies the maximal principle (Definition 3$)$.

Proof Since $\widehat{B}^{\star}$ is one to one, it amounts to check that, if $\widehat{B} U \in-\Lambda_{-}$, i.e. if $U$ is such that

$$
\int_{\Omega} U \cdot \nabla q \geq 0 \quad \forall q \in \Lambda, q \geq 0 \text { a.e., }
$$

then the (unique) solution $p \in \Lambda$ to

$$
\int_{\Omega} \nabla p \cdot \nabla q=\int_{\Omega} U \cdot \nabla q \quad \forall q \in \Lambda
$$

takes nonnegative values almost everywhere, i.e. it lies in $\Lambda_{+}$. This property takes the form of a maximum principle for the Laplace operator, in an extended sense: the saturated zone $[\rho=1]$ may be not be the closure of an open domain, it may in particular have an empty interior, while having a positive measure (see Remark 1). This property is obtained by a standard procedure, which consists in taking a test function equal to the negative part of $p$, i.e. $q=p^{-}=-\min (0, p)$. We have that $\nabla q=-\nabla p \mathbf{1}_{p \leq 0}$ (see Theorem 1.56 in [35]), and $q \geq 0$, so that

$$
-\int_{\Omega}\left|\nabla p^{-}\right|^{2}=\int_{\Omega} U \cdot \nabla p^{-} \geq 0
$$

which implies that $\nabla p^{-}$vanishes almost everywhere, i.e. $p^{-}$is constant on $\Omega$. Since it is 0 in the neighborhood of the boundary, it vanishes on $\Omega$ i.e. $p \geq 0$ a.e. in $\Omega$.

In other words, if the pre-collisional field is non-expansive, i.e. $\nabla \cdot U \leq 0$, then the pressure field $p$ is a weak solution to the Poisson problem

$$
-\Delta p=-\nabla \cdot U,
$$

on the saturated zone $[\rho=1]$, with homogeneous boundary conditions.

Let us add that the PDE above can only we legitimately written under certain conditions on the saturated zone. If the latter presents some pathologies, for example 
if it has an empty interior (like the complement of a dense open set) and yet a positive measure, then $p$ might be non-trivial ( $\Lambda$ is not reduced to $\{0\}$ ), whereas (20) is not even verified in the sense of distributions. Indeed, since $\Lambda$ does not contain any smooth function (except 0), the formulation (19) is much weaker than (20) considered in the sense of distribution.

A proper Poisson problem can be recovered under some additional assumptions, for instance if the saturated zone is "regular" in the sense that $[\rho=1]=\bar{\omega}$ where $\omega$ is a smooth domain. The condition that is actually needed is actually the following: $\omega$ is such that $\Lambda$ (defined by (11)) is equal to the closure of $C_{c}^{\infty}(\omega)$ in $H^{1}(\Omega)$ (the functions of $C_{c}^{\infty}(\omega)$ being extended by 0 outside $\omega$ ). Under these conditions, (19) implies that $p$ is a weak solution (in a standard sense) to the Poisson problem (20).

Remark 1 We mentioned the fact that the space $\Lambda$ might contain no smooth function, while being non-trivial. We prove in this remark that it is indeed the case. We propose to investigate the case where the saturated zone $[\rho=1]$, which contains the support of all those functions in $\Lambda$, is the complement of a dense open set $\omega$, which excludes any nontrivial smooth function. We describe below how to build a nontrivial function in $\Lambda$. We assume $d=2$, but the approach can be straightforwardly extended to higher dimensions. We consider a sequence $\left(c_{n}\right) \in \Omega^{\mathbb{N}}$ that is dense in $\Omega$, and a sequence $\left(R_{n}\right) \in(0,+\infty]^{\mathbb{N}}$ such that $\sum \pi R_{n}^{2} \leq|\Omega| / 2$. For a given $r_{n}<R_{n}$, we denote by $\gamma_{n}$ the circle of radius $r_{n}$, centered at $c_{n}$, by $\Gamma_{n}$ the cocentric circle of radius $R_{n}$, and by $\Omega_{n}$ the ring domain between these circles. We denote by $g_{n}$ the solution to the following Dirichlet problem in $\Omega_{n}$

$$
\mid \begin{aligned}
-\Delta g & =0 \text { in } \Omega_{n}, \\
g & =0 \text { on } \gamma_{n}, \\
g & =1 \text { on } \Gamma_{n},
\end{aligned}
$$

extended by 0 inside the small disc, and by 1 outside the large one. Since the capacity of a point is 0 in $\mathbb{R}^{2}$ (see e.g. [24]), one can choose $r_{n}$, with $0<r_{n}<R_{n}$, sufficiently small to ensure that

$$
\int_{\Omega}\left|\nabla g_{n}\right|^{2} \leq \frac{1}{2^{2 n}}
$$

We denote by $\omega$ the union of the small discs (centered at $c_{n}$, with radius $r_{n}$ ), which is open and dense by construction. Now consider the function $G_{n}=g_{1} g_{2} \ldots g_{n}$. It holds that

$$
\nabla G_{n}=\sum_{k=0}^{n} \nabla g_{n} \sum_{j \neq k} g_{j},
$$

so that (all the $g_{j}$ take values between 0 and 1 by construction), by the triangular inequality in $L^{2}(\Omega)$,

$$
\left\|\nabla G_{n}\right\|_{L^{2}(\Omega)} \leq \sum_{k=0}^{n}\left\|\nabla g_{k}\right\|_{L^{2}(\Omega)} \leq \sum_{k=1}^{n} \frac{1}{2^{k}} \leq 2 .
$$


Fig. 4 In the configuration represented here, considering that the distances are subject to remain 0 (constraint $B u=0$ ), the pre-collisional velocity tends to push any two grains in contact toward overlapping, and yet the pressure between the two grains in the center will be negative.

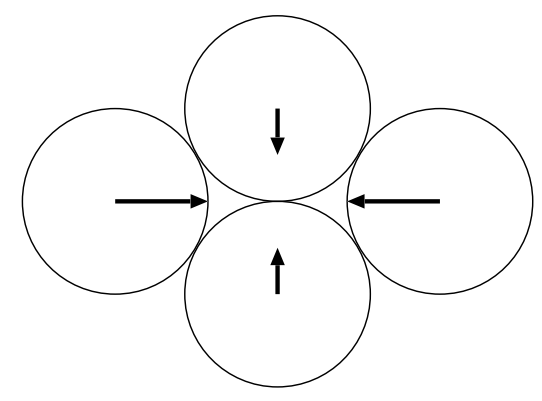

The sequence $\left(G_{n}\right)$ is therefore bounded in $H^{1}(\Omega)$ (the gradient is bounded in $L^{2}$, and they all vanish in the first disc centered at $c_{0}$, with radius $r_{0}$ ). One can extract a sub-sequence which weakly converges in $H^{1}(\Omega)$ to some function $G \in H^{1}(\Omega)$. Since the convergence is strong in $L^{2}$ by Rellich's Theorem, the convergence (up to a subsequence) holds almost everywhere, so that $G$ is by construction equal to 1 almost everywhere in the complement of the union of the large discs (centered at $c_{n}$, with radius $R_{n}$ ). By assumption on $R_{n}$, the measure of this set is positive (larger than $|\Omega| / 2$ ), so that $G$ is different from 0 , while vanishing by construction in the dense union $\omega$ of the small discs.

At the microscopic level the picture is different in general. In particular, the approach carried out in the proof on the previous proposition is no longer valid. The difficulty comes from the fact that $B B^{\star}$, which is the straight analog of $-\Delta$ in the one-dimensional setting, does not verify any maximum principle in general.

Consider the simple situation represented in Figure 4, with a pre-collisional velocity directed toward the center. If we consider (like in the proof of the previous proposition) the problem with an equality constraint $(B u=0$, which means that the hard grains are glued together), eliminating the velocity leads to a discrete Poisson problem

$$
B B^{\star} p=B U .
$$

Since the horizontal velocities have a much larger magnitude, in spite of the fact that the pre-collisional velocity pushes the grains against each other (i.e. $B U>0$ ), it is clear that the pressure associated with the contact between the two central grains will be negative, which rules out the maximum principle (in the sense of Definition 3). As a consequence, the solution to the impact problem, with a unilateral constraint, will not be the same: the grains at the center will be pushed apart during the collision, which implies (thanks to the complementarity constraint $\langle B u \mid p\rangle=0$ ) that the corresponding pressure is 0 . Note that some sort of Poisson Problem can be recovered for the pressure associated with the impact law, by removing the raws of $B$ which correspond to non activated contacts, i.e. with $-G_{i j} \cdot u<0$. If one denote by $\bar{B}$ the corresponding matrix, and by $\bar{p}$ the corresponding pressure, it holds that

$$
\bar{B} \bar{B}^{\star} \bar{p}=\bar{B} \bar{U}
$$


with a reduced matrix $\bar{B}$ which may also not verify the maximum principle.

This violation of the maximum principle for $B B^{\star}$ is generic in the hard-sphere, microscopic, setting, as soon a dense collections of grains are concerned. It can be checked for simple situation that the matrix $B B^{\star}$, unlike in the one-dimensional setting, has positive off-diagonal entries.

\section{Square and triangular lattices}

As an illustration of the previous considerations, and as an introduction to the next section, let us make some remarks on very specific situations, where the overall behavior of a collection of rigid discs can be seen to significantly differ from the behavior given by the macroscopic impact law.

Consider at first a jammed configuration structured according to a square lattice (see Figure 3 (left)). On each row, the non-overlapping constraints impose horizontal velocities to be non-decreasing. Similarly, on each column, the vertical velocities must be non-decreasing also. Two fields of Lagrange multipliers can therefore be associated to the constraints in the main directions $x$ and $y$, which act on the system independently from each other. As a consequence, two constraints must be verified, to be compared to the single scalar constraint of the macroscopic constraint $\nabla \cdot u \geq 0$.

In the case of a triangular lattice (see Figure 3 (right)), the monotonicity of the velocity is imposed in each of the 3 principal directions.

In both cases, the microscopic constraints are much stronger than the macroscopic one, which is therefore obviously irrelevant to model at the macroscopic scale the collections of hard discs. The next section is dedicated to designing macroscopic models more respectful of the underlying microscopic structure, in the case of crystal-like configurations.

\section{Anisotropic macroscopic collision laws}

We develop here some macroscopic models intended to represent configuration of jammed grains introduced in section 3, namely configurations that are structured in a periodic way. The approach is the following: starting from the constraints at the microscopic level, we extend them to the macroscopic level.

\section{Square lattice}

We first propose a macroscopic model adapted from the microscopic configuration of spheres jammed on a cartesian lattice, as depicted on Figure 5. Let us study the constraints on the velocity of the central sphere denoted by 0 in the Figure: there are four, each one corresponding to a contact with an adjacent sphere. The microscopic constraint for spheres in contact described by (2) writes here 

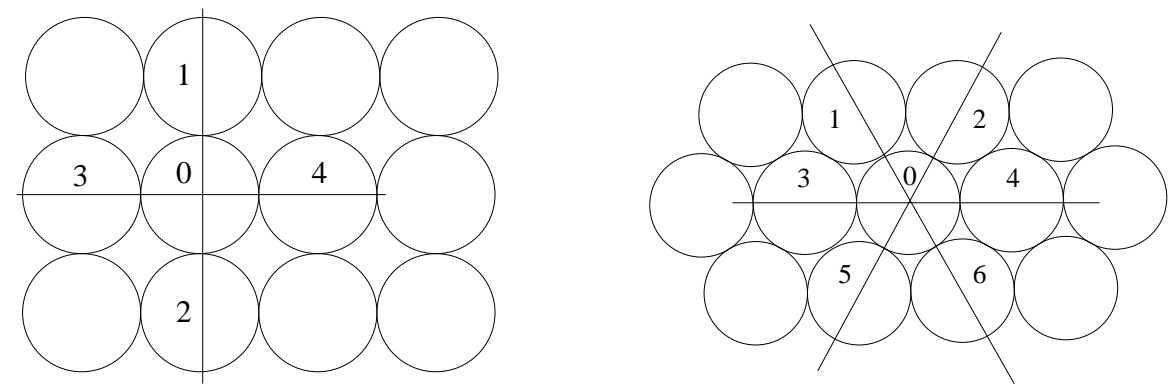

Fig. 5 The two structured jammed configuration. On both side, the spheres in contact with the sphere 0 are labelled from 1 to 4 or 6 .

$$
\mid \begin{aligned}
& \left(u_{1}-u_{0}\right) \cdot e_{y} \geq 0 \\
& \left(u_{0}-u_{2}\right) \cdot e_{y} \geq 0 \\
& \left(u_{4}-u_{0}\right) \cdot e_{x} \geq 0 \\
& \left(u_{0}-u_{3}\right) \cdot e_{x} \geq 0
\end{aligned}
$$

System (22) can be reformulated in a more concise way: the quantities $u_{x}=u \cdot e_{x}$ and $u_{y}=u \cdot e_{y}$ must be non decreasing along each axis. In a macroscopic setting, we want thus to translate this constraint by subjecting $\partial u_{x} / \partial x$ and $\partial u_{y} / \partial y$ to be nonnegative in some sense, considering that $u$ has $L^{2}$ regularity. To that purpose, we define the directional derivatives of the two components in a dual way, imposing

$$
-\int_{\Omega} u_{x} \frac{\partial p}{\partial x} \geq 0
$$

for every nonnegative test function $p$ such that its weak partial derivative in $x$ can be defined. In order to clarify this last condition, we will introduce anisotropic Sobolev spaces, naturally defined to formalize the notion of "weakly derivable along one direction". The following description of these spaces is extracted from [16]. In what follows, $\Omega$ is a strictly convex bounded open set, with regular boundary. We refer to [16] for the study of more general domains.

Definition 1 The anisotropic Sobolev space in the direction $x$ on $\Omega$ is defined by:

$$
H_{x}^{1}(\Omega)=\left\{f \in L^{2}(\Omega), \frac{\partial f}{\partial x} \text { weakly exists in } L^{2}(\Omega)\right\}
$$

where " $\frac{\partial f}{\partial x}$ weakly exists in $L^{2}(\Omega)$ " means that

$$
\forall g \in C^{1}(\Omega), \int_{\Omega} \frac{\partial f}{\partial x} g=-\int_{\Omega} \frac{\partial g}{\partial x} f .
$$

This space is endowed with the norm $\|f\|_{H_{x}^{1}}^{2}=\|f\|_{2}^{2}+\left\|\frac{\partial f}{\partial x}\right\|_{2}^{2}$ 
We then define $H_{0, x}^{1}(\Omega)$ as the closure of $C_{c}^{\infty}(\Omega)$ in $H_{x}^{1}(\Omega)$, and $H_{x}^{-1}(\Omega)$ as the dual of $H_{0, x}^{1}(\Omega)$. Since integrating along a single direction is sufficient to prove Poincaré inequality in the usual Sobolev space $H_{0}^{1}(\Omega)$ (see Proposition 9.18 and Corollary 9.19 in [9]), the anisotropic counterpart of Poincaré inequality holds :

Proposition 5 There exists $c>0$ such that for every $f \in H_{0, x}^{1}(\Omega)$,

$$
\|f\|_{2} \leq c\left\|\frac{\partial f}{\partial x}\right\|_{2}
$$

We may now introduce the macroscopic model corresponding to a square microscopic structure. Let $\rho \in L^{\infty}(\Omega)$, such that $\rho \leq 1$ a.e., we still denote by $\rho$ the measure of density $\rho$ with respect to the Lebesgue measure. In order to avoid boundary issues, we assume that all the measures we consider are supported on a set strongly included in $\Omega$ (that is to say, as previously, at positive distance to $\partial \Omega$ ). In order to alleviate notation (and to deal with realistic situations when the grains configuration is structured), we assume $\rho$ to be saturated over all its support, so that there is no need in mentioning the dependence in $\rho$ in the notation.

Problem 1 Given $\rho \leq 1$ and $U \in L^{2}(\Omega)^{2}$, find $u=\left(u_{x}, u_{y}\right) \in L^{2}(\Omega)^{2}$ that realizes the projection

$$
\min _{u \in C^{x} \cap C^{y}} \int_{\Omega}\|u-U\|_{2}^{2} d \rho
$$

where the constraints set $C^{x}$ and $C^{y}$ are defined by duality:

$$
C^{\alpha}=\left\{u \in L^{2}(\Omega), \int_{\Omega} \frac{\partial q}{\partial e_{\alpha}} u_{\alpha} \leq 0 \quad \forall q \in \Lambda_{\alpha}, q \geq 0 \quad \text { a.e }\right\}
$$

and $\Lambda_{\alpha}=\left\{q \in H_{0, e_{\alpha}}^{1}(\Omega), q(1-\rho)=0 \quad\right.$ a.e. $\}$ for $\alpha=x, y$.

Since $C^{x}$ and $C^{y}$ are closed convex cones, the projection problem 1 admits a unique solution. We can write the saddle-point formulation of the problem, that is the instantiation of the abstract formulation (50) to the present situation.

Proposition 6 There exists a unique pair of nonnegative Lagrange multipliers (or pressures) $\left(p_{x}, p_{y}\right) \in \Lambda_{x} \times \Lambda_{y}$ such that

$$
u+\frac{\partial p_{x}}{\partial x} e_{x}+\frac{\partial p_{y}}{\partial y} e_{y}=U \text { o-a.e. }
$$

Proof The constraint set $C=C^{x} \cap C^{y}$ can be written

$$
C=\left\{u \in L^{2}(\Omega)^{2},\left\langle B u \mid\left(q_{x}, q_{y}\right)\right\rangle \leq 0, \forall\left(q_{x}, q_{y}\right) \in \Lambda_{+}\right\}
$$

where 


$$
B:\left\{\begin{aligned}
L^{2}(\Omega)^{2} & \longrightarrow H_{x}^{-1}(\Omega) \times H_{y}^{-1}(\Omega) \\
u & \longmapsto\left(-\frac{\partial u_{x}}{\partial x},-\frac{\partial u_{y}}{\partial y}\right)
\end{aligned}\right.
$$

and $\Lambda_{+}=\left\{\left(q_{x}, q_{y}\right) \in \Lambda_{x} \times \Lambda_{y}, q_{x} \geq 0, q_{y} \geq 0 \quad\right.$ a.e. $\}$. By Poincaré inequality, there exists a constant $\beta>0$ such that $\left|B^{\star} \mu\right| \geq \beta|\mu|$, with

$$
B^{\star}:\left\{\begin{aligned}
H_{0, x}^{1}(\Omega) \times H_{0, y}^{1}(\Omega) & \longrightarrow L^{2}(\Omega)^{2} \\
\left(q_{x}, q_{y}\right) & \longmapsto \frac{\partial q_{x}}{\partial x} e_{x}+\frac{\partial q_{y}}{\partial y} e_{y} .
\end{aligned}\right.
$$

Corollary 1 in the appendix guarantees the existence of a pair of Lagrange multipliers, the uniqueness comes from the one-to-one character of $B^{\star}$ given by the same inequality.

The saddle-point formulation (29) can be projected onto the two axes, leading to two independant systems. The problem then reduces to finding

$$
\left(u_{x}, p_{x}\right) \in L^{2}(\Omega) \times H_{0, x}^{1}(\Omega) \text { and }\left(u_{y}, p_{y}\right) \in L^{2}(\Omega) \times H_{0, y}^{1}(\Omega)
$$

solutions to

$$
\begin{aligned}
& \frac{\partial p_{x}}{\partial_{x}}+u_{x}=U_{x} \quad \rho \text {-a.e. } \\
& -\frac{\partial u_{x}}{\partial x} \quad \leq 0 \text { where } \rho=1, \\
& \mid \begin{array}{l}
\frac{\partial p_{y}}{\partial y}+u_{y}=U_{y} \quad \rho \text {-a.e. } \\
-\frac{\partial u_{y}}{\partial y} \quad \leq 0 \quad \text { where } \rho=1 .
\end{array}
\end{aligned}
$$

This is the macroscopic counterpart of what we had seen at the end of Section 3: two independant pressure fields appear, acting separately on each component of the velocity in order to correct the compressions in $x$ and $y$.

Remark 2 This model introduces anisotropy, so that the collision is no longer rotationally invariant: Figure 6 shows the situation of two colliding blocks, under three angles of impact. In the case of an impact along one of the two principal directions, no perturbation occurs in the tangential direction whereas in the case of an impact involving both directions (second case in Figure 6), a transverse velocity appear. Note that in the third case of two blocks colliding along a direction that is very close to one of the two axes, the post-impact velocity is mainly directed along the transverse direction.

We shall now build a more pathological macroscopic model derived from the microscopic configuration of a triangular (or hexagonal) stack of particles (see Figure 5, right). The well-posedness of the saddle-point formulation (i.e. existence 

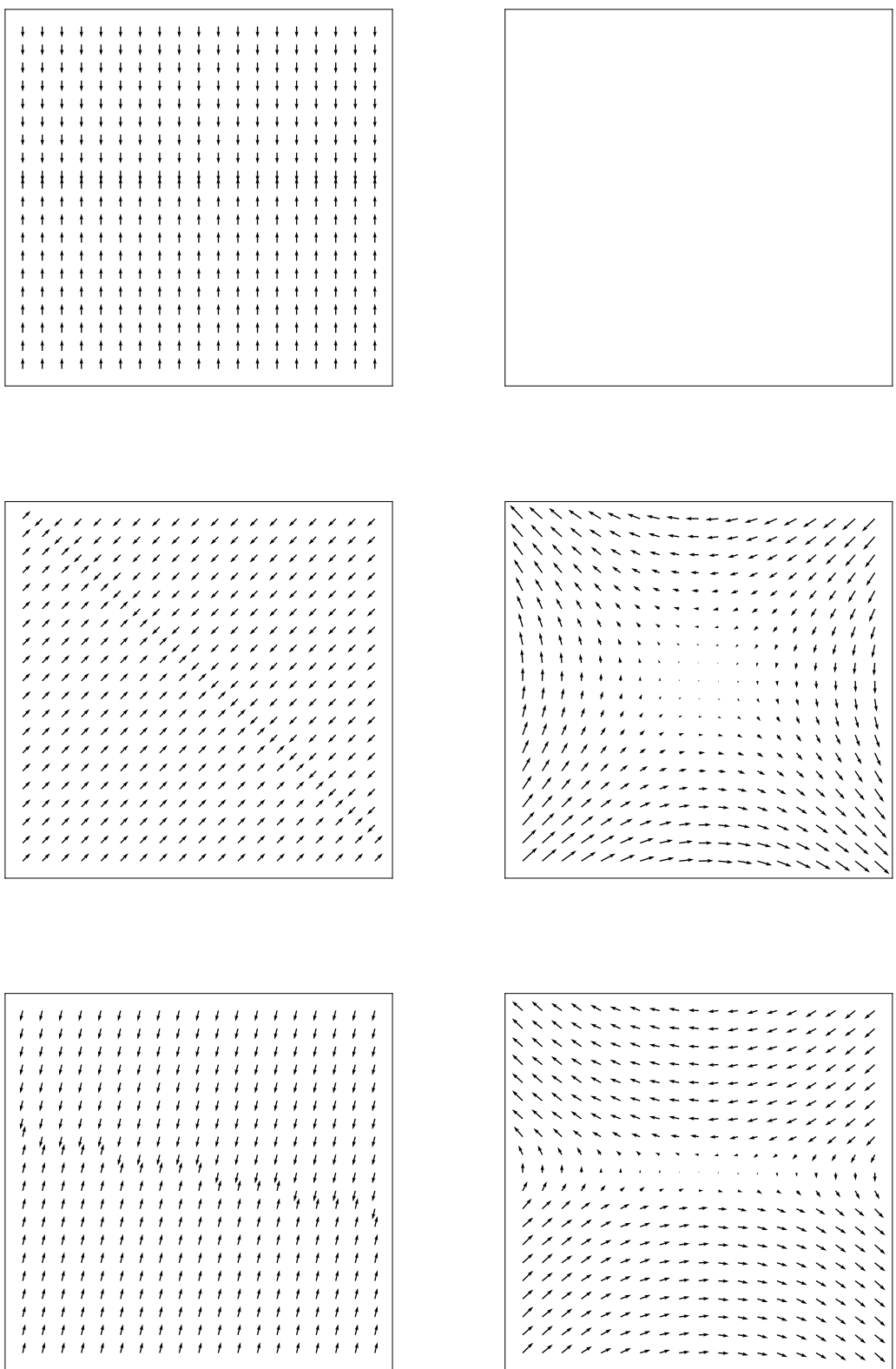

Fig. 6 Three impacts between opposing blocks, varying the angle of incidence. On the left, the velocity fields before the impact; on the right, the velocity fields after the impacts. 
and uniqueness of pressure fields) is more delicate than before: uniqueness is lost, as we shall see later on, and the existence is still an open problem. We introduce unit vectors along the principal directions:

$$
e_{0}=(1,0), e_{1}=\left(-\frac{1}{2}, \frac{\sqrt{3}}{2}\right), e_{2}=\left(-\frac{1}{2},-\frac{\sqrt{3}}{2}\right) .
$$

In this case, any sphere has 6 neighbors, two along each axis directed by the $e_{i}$. As for the previous configuration, one can write the microscopic constraints on the sphere 0

$$
\mid \begin{aligned}
& \left(u_{4}-u_{0}\right) \cdot e_{0} \geq 0,\left(u_{0}-u_{3}\right) \cdot e_{0} \geq 0,\left(u_{1}-u_{0}\right) \cdot e_{1} \geq 0 \\
& \left(u_{0}-u_{6}\right) \cdot e_{1} \geq 0,\left(u_{5}-u_{0}\right) \cdot e_{2} \geq 0,\left(u_{0}-u_{2}\right) \cdot e_{2} \geq 0
\end{aligned}
$$

that can be reformulated by saying that $u_{i}=u \cdot e_{i}$ has to be increasing along each axis of constraint. We can thus write the macroscopic model reformulating this monotonicity exactly as above: the non-overlapping constraint becomes $B u \leq 0$, with

$$
B:\left\{\begin{aligned}
L^{2}(\Omega)^{2} & \longrightarrow H_{e_{0}}^{-1}(\Omega) \times H_{e_{1}}^{-1} \times H_{e_{2}}^{-1}(\Omega) \\
u & \longmapsto\left(-\partial_{e_{0}} u_{0},-\partial_{e_{1}} u_{1}, \partial_{e_{2}} u_{2}\right)
\end{aligned}\right.
$$

where $u_{i}$ is the projection of $u$ on the vector $e_{i}$. The triangular macroscopic model then writes

Problem 2 Given $\rho \leq 1$ and $U \in L^{2}(\Omega)^{2}$, find $u \in L^{2}(\Omega)^{2}$ that realizes the projection

$$
\min _{B u \leq 0} \int_{\Omega}\|u-U\|_{2}^{2} d \rho
$$

The constraint $B u \leq 0$ is to be interpreted in a dual way: we then require $\langle B u \mid q\rangle \leq 0$ for every nonnegative pressures $q \in \Lambda_{0} \times \Lambda_{1} \times \Lambda_{2}$, with

$$
\Lambda_{i}=\left\{q_{i} \in H_{0, e_{i}}^{1}(\Omega), q(1-\rho)=0 \quad \text { a.e. }\right\}, i=0,1,2 .
$$

Since Problem 2 consists in projecting on a closed convex cone, it admits a unique solution. The saddle-point formulation reads:

$$
\begin{aligned}
& \text { Find }\left(u, p_{0}, p_{1}, p_{2}\right) \in\left(L^{2}(\Omega)^{2} \times \Lambda_{0} \times \Lambda_{1} \times \Lambda_{2}\right) \text { such that } \\
& \qquad \begin{array}{l}
u+\sum_{i=0}^{2} \partial_{e_{i}} p_{i} e_{i}=U, \\
p_{i} \geq 0 \\
\sum_{i=0}^{2} \int_{\Omega} u_{i} \partial_{e_{i}} p_{i}=0 .
\end{array}
\end{aligned}
$$


Remark 3 The operator $B^{\star}$ is not onto, as we show here by exhibiting a non-trivial element in $\operatorname{ker}\left(B^{\star}\right)$. Consider an hexagon $H$ included in $\Omega$, and $f$ a piecewise constant function equal to 0 out of $H$, and alternatively 1 and -1 depending on the position in $H$, as represented on Figure 7. Define now $p_{i}$ as the solution of

$$
\mid \begin{array}{cc}
\partial_{i} p_{i}=f & \text { in } H \\
p_{i}=0 & \text { in } \Omega \backslash H
\end{array}
$$

Due to the symmetries of $f$, this equation is compatible with the limit condition $p=0$ on $\partial H$ : every line directed by any $e_{i}$ has an intersection of the same length with zones labelled by 1 or -1 . Moreover, we have $\sum_{i=0}^{2} \partial_{i} p_{i} e_{i}=f \sum_{i=0}^{2} e_{i}=0$, so $p$ lies in $\operatorname{ker}\left(B^{\star}\right)$.

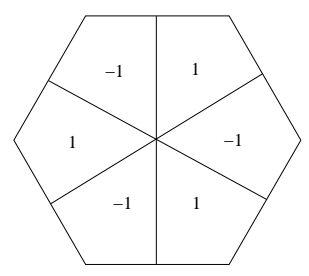

Fig. 7 Counterexample to the injectivity of $B^{\star}$

Remark 4 It is an interesting counterpart to the microscopic counterexample presented on Figure 2. When the number of spheres increases, there is $3 / 2$ times more constraints than degrees of freedom; thus the dimension of the kernel of $B^{\star}$ tends to infinity in the micro case. Accordingly, the macroscopic example above provides an infinite family of independent vectors in $\operatorname{ker}\left(B^{\star}\right)$.

\section{Homogenization issues}

This section deals with issues pertaining to the convergence of microscopic models towards macroscopic ones. Let us make it clear that such convergence is out of reach in general. We shall rather describe a general framework to address these issues, and establish some convergence results in very particular situations, in the case when the microscopic situation is structured. The idea is the following: we start from a macroscopic velocity field, and we span the domain with a sequence of saturated configurations of spheres of radius tending to 0 . At each scale, we project the field on the feasible set, which contains all those fields which comply with the non-overlapping constraint. 

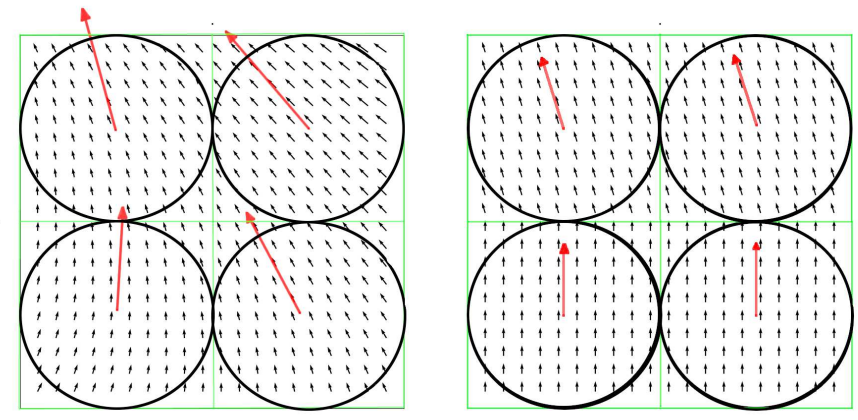

Fig. 8 On the left, the construction of a microscopic vector field $\tilde{U}$ from the macroscopic one $U$, in the square configuration. The green lines delimit the Voronoï cells associated to the spheres, the red arrows are the mean of the vector field on each cell (expanded for a sake of clarity). On the right, construction of a macroscopic vector field $v$ (black) from a microscopic field $\tilde{u}$ (red).

\subsection{General procedure}

We describe here a general procedure to formalize questions concerning micro-macro convergence. First, we need to define a way to compare microscopic velocities to macroscopic fields. Given a field $U \in L^{2}(\Omega)^{2}$, and $n$ non overlapping touching spheres in $\Omega$, denote $D_{1}, \ldots, D_{n} \subset \Omega$ the Voronoï cells associated to the spheres. Define

$$
\tilde{U}_{i}=\frac{1}{\left|D_{i}\right|} \int_{D_{i}} U(x) d \rho(x) \quad \forall 1 \leq i \leq n .
$$

Let $\tilde{u}$ be the solution of the microscopic problem associated to $\tilde{U}$. Finally, let $v$ be the piecewise constant function equal to $\tilde{u}$ on the cell $D_{i}$. This mapping is depicted in Figure 8. We have built an operator

$$
\phi_{n}:\left\{\begin{aligned}
L^{2}(\Omega)^{2} & \longrightarrow L^{2}(\Omega)^{2} \\
U & \longmapsto v
\end{aligned}\right.
$$

which maps a pre-collisional macroscopic velocity $U$ to a post-collisional velocity, computed through projection at the microscopic level.

We are now able to formulate the homogenization problem statement, in terms of two general questions:

\section{? Homogenization of impact laws}

Given a velocity field $U \in L^{2}(\Omega)^{2}$ and a sequence $\left(x^{n}\right)=\left(x_{i}^{n}\right)_{1 \leq i \leq n}$ of collections of hard sphere configurations, with a common radius $\delta_{n}$ (with $\delta_{n} \rightarrow 0$ ), such that 


$$
\sum_{i=1}^{n} \mathbf{1}_{B\left(x_{i}^{n}, \delta_{n}\right)}
$$

weakly converges to some limit density when $n$ goes to $+\infty$, what are the possible limits of $\phi_{n}(U)$ ? Is it possible to prescribe constraints on the microscopic structure so that $\phi_{n}$ converges to some projection operator at the macroscopic level, which would encode the characteristics of the microscopic structure?

These questions should be seen as a wide research program which is way beyond the scope of these notes. We shall restrict ourselves to some short comments, and to providing a detailed answer in very specific situations (see Section 5.2).

First, various sorts of constraints can be expected: isotropic ones like in Section 2, anisotropic ones according to some principal directions like in Section 4, or possibly not linked to any underlying regular structure in the grain configuration. The notion of local maximal value, already discussed in Section 2, is also an issue: consider e.g. a configuration where a part of the saturated domain is spanned by a square lattice, and another part is spanned by a triangular mesh. As we shall see below, the projection operators will actually converge towards an operator activated respectively when $\rho=(1-\pi / 4)$ and $\rho=\pi / 2 \sqrt{3}$, depending on the local microscopic structure, so that the maximal density is not defined uniformly over the saturated zone.

In all generality, when there is no reason to assume any regularity / periodicity in the microscopic structure, one may expect some sort of averaging in the direction of contacts, with a local constraint on the density based on the so-called Random Maximal Packing, that is around 0.64 for three-dimensional collections of identical hard spheres ([33]). This may legitimate an isotropic approach like the one presented in Section 2.2, based on a uniform maximal density, and an isotropic constraint on the velocities. Yet, as extensively described in the literature on granular media (see e.g. [32]), complex force networks are observed within collections of grains, over scales that go way beyond the microscopic size of the grains. Such observations advocate for the need to develop macroscopic models which would reflect some anisotropy at the mesoscopic scale, in the spirit of what is done in the next section for highly structured configurations.

Remark 5 One could question the choice of using Voronoï Cells instead of defining the field $v$ to be constant on every sphere, and null elsewhere. The reason is that we aim at showing strong $L^{2}$ convergence results, which will not hold for velocities supported on spheres. For instance, consider the constant field $U=e_{x}$ for the squared configuration with radius tending to 0 . As no constraint is activated, $v$ is automatically equal to $U$ everywhere, and similarly, $\tilde{u}_{i}^{n}=e_{x}$ for every $1 \leq i \leq k_{n}\left(k_{n}\right.$ being the number of spheres needed to span the domain). If we define $w^{n}$ piecewise constant on every sphere equal to 0 elsewhere, there subsists an irreducible gap

$$
\left\|U-w^{n}\right\|_{2}^{2}=(1-\pi / 4) \lambda(\Omega)+o(1)
$$

$\pi / 4$ being the proportion of $\Omega$ spanned by the spheres for a square lattice. 


\subsection{Homogenization for structured configurations}

We detail here micro-macro convergence results in very specific situations, namely when the microscopic spheres on which we interpolate in the previous subsection are organized on square or triangular lattices. More precisely, under the framework presented at the beginning of Section 5.1, we establish that, given a pre-collisional velocity field $U$, the velocities obtained by projection at the microscopic level (operators $\phi_{n}$ defined by (38)) converge to a velocity obtained by projection at the macroscopic level, according to the projection operators detailed in Section 4. Here, for the sake of simplicity we consider the whole set $\omega=[0,1] \times[0,1]$ to be spanned by spheres disposed on a square or triangular lattice, as in Figure 5. We can thus disregard the issues of maximal density raised in the previous section, as we know that the microscopic density measure will weakly converge to a constant, that we can set to 1 .

First, we need to fix some common notation for the two structures considered. Let $V=L^{2}(\omega)^{2}$ be the set of macroscopic velocities, and for $n \in \mathbb{N}, k_{n}$ the number of spheres of radius $1 / n$ needed to span $\omega$, either for the square or the triangular configuration. Denote then $\tilde{V}_{n}=\left(\mathbb{R}^{2}\right)^{n}$ the set of microscopic velocities, $V_{n} \subset V$ the set of functions constant on each Voronoï cell $D_{i} \subset \omega$ associated to the sphere configuration.

We are going to use a classical theorem in Numerical Analysis, designed to estimate errors for approximated problems of optimisation. Here $V_{n}$ is seen as an approximation space for $V$. The main idea here is to approximate not only the space of functions $V$, but also the space of constraints. We denote by $C_{\rho} \subset V$ the set of velocities satisfying the macroscopic anisotropic constraint (defined in section 4), and $C_{n} \subset V_{n}$ the set of velocities satisfying the microscopic constraint once the velocity of a cell is attributed to the sphere in the cell: $C_{n}$ is seen as an approximation of the constraint space $C_{\rho}$. We then have the following error estimate between the exact solution $u$ and the approximate solution $v$ :

\section{Theorem 1 ( Adapted from Falk, 74' [13])}

There exists $a, b>0$ such that for every $f \in C_{n}$ and $g \in C_{\rho}$,

$$
\|u-v\|_{2} \leq\left(a\|u-f\|_{2}^{2}+b\|u-U\|_{2}\left(\|u-f\|_{2}+\|g-v\|_{2}\right)\right)^{1 / 2}
$$

Given $U \in V$, denote by $\tilde{U}^{n}$ the approximation defined by (37) and $v_{n}=\phi_{n}(U)$. Denote as before $u$ and $\tilde{u}^{n}$ the solutions of the macroscopic and the microscopic problems associated to $U$ and $\tilde{U}^{n}$, with respect to the constraint sets $C_{\rho}$ and $C_{n}$. Figure 8 illustrates this construction.

Proposition 7 The sequence $v_{n}$ converges towards $u$ in $L^{2}(\omega)^{2}$.

Proof Let $u_{n}^{\text {int }}$ be the piecewise constant function equal to $\frac{1}{\left|D_{i}\right|} \int_{D_{i}} u(x) d x$ on every $D_{i}$. Since $u$ is in $C_{\rho}$, one can verify that $u_{n}^{i n t} \in C_{n}$. On the other hand, since $\tilde{u}^{n}$ is the 
solution to the microscopic problem, $v_{n}$ is in $C_{\rho}$. Therefore, we can use Theorem 1, that guarantees the existence of $a, b$ some positive constants such that for every $n \in \mathbb{N}$

$$
\left\|u-v_{n}\right\|_{2}^{2} \leq a\left\|u-u_{n}^{i n t}\right\|_{2}^{2}+b\|u-U\|_{2}\left\|u-u_{n}^{i n t}\right\|_{2}
$$

It is then sufficient to show that the piecewise constant approximation $u_{n}^{\text {int }}$ tends to $u$ in $L^{2}(\omega)^{2}$. Let $\epsilon>0$ and $f \in C^{0}(\omega)^{2}$ be such that $\|u-f\|_{2} \leq \epsilon$; denote $f_{n}^{\text {int }}$ the piecewise constant approximation of $f$. We have

$$
\left\|u-u_{n}^{i n t}\right\|_{2} \leq\|u-f\|_{2}+\left\|f-f_{n}^{i n t}\right\|_{2}+\left\|f_{n}^{i n t}-u_{n}^{i n t}\right\|_{2}
$$

Using that $f$ is uniformly continuous on $\omega$, define $n_{0} \in \mathbb{N}$ such that for every $x, y \in \omega$ satisfying $|x-y| \leq \frac{2}{n_{0}},|f(x)-f(y)| \leq \epsilon$. Thus for $n \geq n_{0}$

$$
\begin{aligned}
\left\|f-f_{n}^{i n t}\right\|_{2}^{2} & \leq \sum_{i=1}^{k_{n}} \int_{D_{i}}\left|f(x)-f_{n}^{i n t}(x)\right|^{2} d x \\
& \leq \sum_{i \in I} \int_{D_{i}} \epsilon^{2}=\epsilon^{2} .
\end{aligned}
$$

On the other hand, using Jensen inequality

$$
\begin{aligned}
\left\|u_{n}^{i n t}-f_{n}^{i n t}\right\|_{2}^{2} & =\sum_{i=1}^{k_{n}} \int_{D_{i}}\left|u_{n}^{i n t}(x)-f_{n}^{i n t}(x)\right|^{2} d x \\
& =\sum_{i=1}^{k_{n}} \lambda\left(D_{i}\right)\left|\frac{1}{\lambda\left(D_{i}\right)} \int_{D_{i}}(u(y)-f(y)) d y\right|^{2} \\
& \leq \sum_{i=1}^{k_{n}} \int_{D_{i}}|u(y)-f(y)|^{2} d y \\
& =\|u-f\|_{2}^{2}
\end{aligned}
$$

Thus $u_{n}^{\text {int }}$ converges in $L^{2}(\omega)^{2}$ towards $u$, and so does $v_{n}$.

Remark 6 In the previous proof, two ingredients can be identified as essential in the process of elaborating general homogenization results:

- $u_{n}^{\text {int }} \in C_{n}$ : a field that respects the macroscopic constraint must check the microscopic constraints once integrated on the Voronoï cells; and reciprocally the piecewise constant approximation $v_{n}$ of the corrected microscopic field must satisfy the macroscopic constraint. Thus the macro/micro constraints must be compatible under the mapping that we defined above.

- $u_{n}^{\text {int }}$ needs to converge for the $L^{2}$ norm toward $u$ : this is in particular true if the spheres span the whole saturation area, in the sense that the diameter of the Voronoï cells tends to 0 . 


\section{Evolution models}

We describe here the evolution problems which are associated to the impact laws that have been described in the first sections of these notes. Let us first make it clear that writing an evolution problem associated to the impact laws studied in Sections 4 and 5 is irrelevant a priori. Indeed, the assumptions which can be made on the microscopic structure of a granular medium are instantaneously ruled out as soon as the medium undergoes any deformation. A macroscopic model respectful of the current state of the medium in terms of microscopic structure should rely on some parameters to reflect the local organization of grains, which strongly conditions the impact law as we detailed in the previous sections. We shall rather present evolution problems for the microscopic setting, which takes the form of a second-order in time differential inclusion, and for the macroscopic scale we shall consider the isotropic setting only (the divergence is nonnegative on the saturated zone).

\section{Microscopic evolution problem}

Like in Section 2.1, we consider $n$ moving rigid spheres centered at $r_{1}, \ldots, r_{n}$, with common radius $R$, subject to forces $f=\left(f_{1}, \ldots, f_{n}\right)$. We denote by $m_{1}, \ldots, m_{n}$, the masses of the grains, and by $M \in \mathcal{M}_{n d}$ the associated mass matrix. We denote by $K$ the feasible set (defined by (5)), by $C_{r}$ the cone of feasible direction (defined by (7)), and by $N_{r}=C_{r}^{\circ}$ the outward normal cone. Note that $N_{r}$ is $\{0\}$ as soon as $r$ lies in the interior of $K$, i.e. when there is no contact. We shall consider ${ }^{1}$ that $N_{r}=\emptyset$ for $r \notin K$. The most concise way to write a class of evolution problems for this system, considering that impacts are frictionless, is the following (see e.g.[30]):

$$
M \frac{d^{2} r}{d t^{2}}+N_{r} \ni f
$$

When there is no contact, $N_{r}=\{0\}$, and we recover $n$ independent ODE's in $\mathbb{R}^{d}$. The contraint $r \in K$ is implicitly prescribed because $N_{r}=\emptyset$ as soon as $r \notin K$. Note also that we have (see the proof of Proposition 1)

$$
N_{r}=\left\{-\sum_{i j} p_{i j} G_{i j}, p_{i j} \geq 0,\right\}
$$

where $G_{i j}$ is defined by (6). It guarantees that contact forces verify the Law of Action-Reaction, and that only repulsive forces are exerted (grains do not glue to each other).

Yet, Inclusion (43) is essentially compatible with all impact laws which do not violate the Law of Action-Reaction, including some laws which would lead to an

1 This convention is consistent with the definition of $N_{r}$ as the Fréchet subdifferential of the indicatrix function $I_{K}$ of $K$, which is indeed $\emptyset$ outside of $K$. 
increase of kinetic energy. An impact law of the type (3) has to be prescribed. We shall now write the full evolution system, in the purely inelastic setting, and with an explicit involvement of interaction forces. In the dynamic setting, these forces are generically singular in order to instantaneously change the velocities of the grains, and we shall represent them by positive measures in time, denoted by $\mathcal{M}_{+}(0, T)$. In the purely inelastic setting, the system writes

$$
\mid \begin{array}{ll}
M \frac{d^{2} r}{d t^{2}} & =f+\sum_{i j} p_{i j} G_{i j} \\
p_{i j} & \in \mathcal{M}_{+}(0, T) \\
\operatorname{supp}\left(p_{i j}\right) & \subset\left\{t, D_{i j}(r(t))=0\right\} \\
u^{+} & =P_{C_{r}} u^{-} .
\end{array}
$$

More general impact laws can be considered, by setting $u^{+}=u^{-}-(1+e) P_{N_{r}} u^{-}$for $e \in[0,1]$. As detailed in $[3,18]$, the relevance of the impact law is ensured by the fact that the velocity has bounded variations in time, so that it admits at each time left and right limits.

The system is formally well-posed in the sense that it fits in classical CauchyLipschitz theory when there is no contact, and whenever a contact occurs the impact law univocally expresses the post-collisional velocity with respect to the precollisional one. It can be checked that kinetic energy is preserved for $e=1$, and part of it is lost during each collision for $e<1$.

There is indeed a well-posedness results for this system, under the condition that the forcing term $f$ is analytic (see e.g. [3]). Counter-examples to uniqueness exist for the case of a single grain and a wall, in the elastic setting $(e=1)$, with a forcing term which is infinitely differentiable (see [30]).

A similar counter-example can be built in the purely non-elastic case $(e=0)$, we again refer to $[30,3]$ for the analytic expression of the forcing term. In order to illustrate the principle of these counter-examples, we plot in Figure 9 a numerical computation of two distinct solutions associated to the same forcing term, for a single particle forced toward a wall. As detailed in [18], the plotted numerical solutions correspond to two different sequences of time steps.

The macroscopic counterpart of (45) is the so-called Pressureless Euler equations with maximal density constraint, which describes the motion of a granular fluid made of particles which do not interact unless saturation (set at $\rho=1$ ) is reached. In the purely inelastic setting, the system writes 

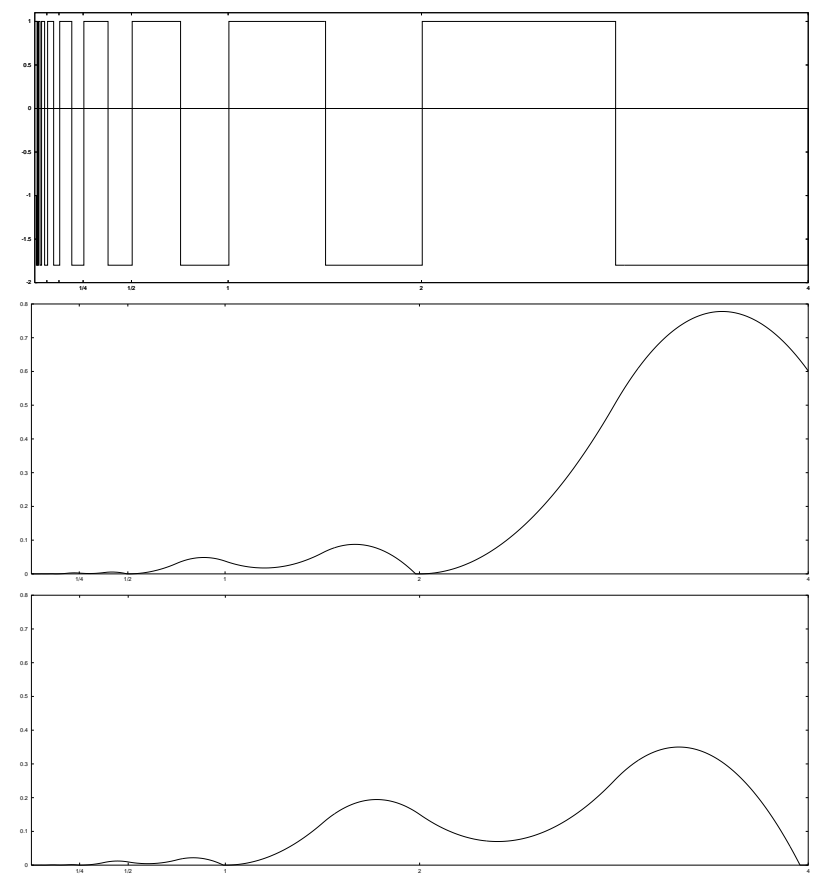

Fig. 9 Two distinct solutions associated to the same forcing term

$$
\left\{\begin{array}{l}
\partial_{t} \rho+\nabla \cdot(\rho u)=0 \\
\partial_{t}(\rho u)+\nabla \cdot(\rho u \otimes u)+\nabla p=0 \\
\rho \leq 1 \\
(1-\rho) p=0 \\
p \geq 0 \\
u^{+}=P_{\widehat{C}_{\rho}}\left(u^{-}\right) .
\end{array}\right.
$$

where $\widehat{C}_{\rho}$ is the cone of feasible velocities defined by (10). These equation must be understood in a weak sense. In particular the pressure $p$ is likely to be very singular in time, like in the microscopic setting, and the momentum equation is meant in a distributional sense. Little is known concerning this system, which is usually written without the impact law (last equation of the system). Note that this law can be replaced, at least formally, by any law of the type

$$
u^{+}=u^{-}-(1+e)\left(u^{-}-P_{\widehat{C}_{\rho}} u^{-}\right),
$$

with $e=1$ for the elastic case. This equation is well-understood in the onedimensional setting, see e.g.[7] where particular "sticky-blocks" solutions are built, and can be used to build solutions of the system. This class of solutions corresponds 
to the situation where the initial density is the sum of characteristic functions of segments, each one initially moving at a uniform velocity. Since no forcing term is involved, segments remains segments, possibly merging to form larger segments, and the model can be treated exactly according to the microscopic model (45). Note that this approach, presented in the purely inelastic setting, could be extended to various impact laws $(e \in(0,1])$. Note also that, since sticky blocks reproduce the microscopic setting, the non-uniqueness result which we mentioned obviously extends to the macroscopic problem, if one accounts for a time-dependent forcing term.

This constructive approach does not straightforwardly extend to higher dimensions for obvious reasons: the saturated zone is likely to deform in a complex way, which makes the macroscopic model fully different from the microscopic one. An extension has nevertheless been proposed recently in [5] to build solutions to (46) (without the impact law), again in a purely non-elastic spirit. A numerical approach is proposed in [10] to approximate candidate solutions to (46). It is based on barotropic Euler equations, i.e. compressible Euler equations where the pressure is assumed to be a function of the local density of barrier type: it is taken in the form $p=\varepsilon p(\rho)$, where $p$ is smooth on $[0,1)$ and blows up to $+\infty$ at $1^{-}$. When $\varepsilon$ goes to 0 , the action of the pressure disappears in non saturated zones, whereas the blow-up at 1 prevents the density to pass the maximal value. Again, the impact law is not integrated in the global limit system, but this approach natively recovers the elastic setting $(e=1)$. In [11], a similar approach is carried out in the case of a variable congestion (the constraint $\rho \leq 1$ is replaced by $\rho \leq \rho^{\star}$, where $\rho^{\star}$ is a given, non-uniform, barrier density). We also refer to [28] for an analysis of a similar system with additional memory effects induced by the presence of an underlying viscous fluid. An alternative approach, also of the constructive type, is proposed in [20], it is based on a time discretization scheme of the splitting type: at each time step the density is transported according to the pre-collisional velocity (the congestion constraint is disregarded), possibly leading to a violation of the constraint. The density is then projected on $\widehat{K}$ according to the Wasserstein distance (like in the crowd motion model presented in $[22,23])$, and the post-collisional velocity is then a posteriori built from the projected density. This approach natively restricts to the purely non-elastic setting. Let us add that these exploratory approaches do not provide a full theoretical framework to the full system (46) (including the impact law).

Let us add a few comments on the difficulty to handle the collision law, in the process of building solutions to the full system. The impact law (last equation of (46)) implicitly assumes that left and right limits exist for the velocity field, which is far from being obvious. In the microscopic setting, it is linked to the BV regularity in time of the velocity field, which makes clear sense in this purely Lagrangian setting. In the purely non-elastic setting the velocity of a given particle may undergo jumps, but each of these jumps also corresponds to a decrease of the kinetic energy. If the forcing term is controlled, the total variation due to these jumps can be shown to be bounded. In the macroscopic setting, the velocity field is defined in a Eulerian way, i.e. $u(x, t)$ corresponds to the current velocity of the medium at $x$, and BV character of velocities for Lagrangian particles has no clear counterpart in this Eulerian description. 

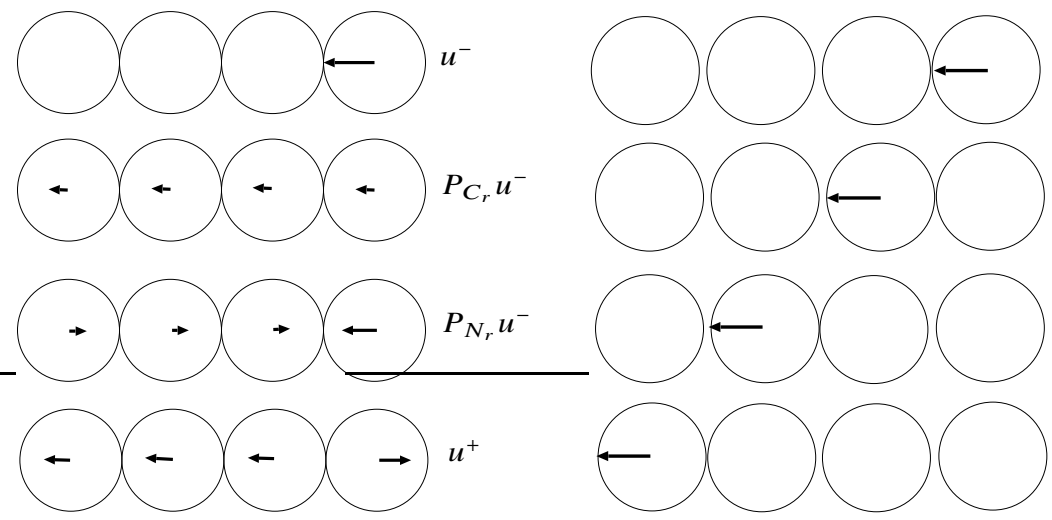

Fig. 10 Newton's craddle: computation of $u^{+}$with Moreau's approach, with initially touching discs (left), and slightly pulled apart discs (right).

\section{Stability issues}

As suggested by the non-uniqueness result for the evolution problems, the problem is unstable with respect to data, and in particular to grain positions. A striking illustration of this instability is given by the so-called Newton's craddle, which can be described as follows: a straight raw of touching identical hard spheres is hit on one of its end by a hard sphere. Actual experiments on this setting show that the apparent post-collisional velocity affects the sphere on the opposite side only, while the other spheres (including the hitting one) stay still. A straight application of the approach we presented (Moreau's approach) in the elastic setting leads to a fully different picture, presented in Figure 10 (left): the hitting sphere is pushed backward (i.e. rightward), almost as if it had hit a wall (the speed is slightly reduced), while the rest of the spheres are pushed leftward at a small velocity, in such a way that total momentum and kinetic energy are conserved. Yet, by considering an initial situation where grains are slightly pulled apart (initial distances set at an arbitrary small value), the experimentally observed behavior is recovered, after a series of quasi-simultaneous binary collisions as illustrated again in Figure 10 (right). Similar examples of the high sensitivity of the impact law to the configuration, possibly inducing significant changes in the future behavior of the system, can straightforwardly be built for the macroscopic one-dimensional problem, in the elastic setting.

\section{Appendix}

We gather here some well-known theoretical results, and some less classical ones, on the saddle-point formulation of cone-constrained minimization problems. 
Let $V$ be a Hilbert space, and $J: V \longrightarrow \mathbb{R}$ a continuously differentiable functional. We denote by $D J(u) \in V^{\prime}$ its differential at $u$, and by $\nabla J(u)$ its gradient:

$$
J(u+h)=J(u)+\langle D J(u), h\rangle+o(h)=J(u)+\langle\nabla J(u) \mid h\rangle+o(h) .
$$

\section{Linear constraints}

Proposition 8 Let $K$ be a linear subspace of $V$, and $u$ a local minimizer of $J$ over $K$. Then $\nabla J(u)$ is orthogonal to $K$, which we can write

$$
\nabla J(u)+\xi=0, \xi \in K^{\perp} .
$$

Proof Fix any $h \in K$. For $t \in \mathbb{R}$ in a neighborhood of 0 ,

$$
J(u+t h)=J(u)+t\langle\nabla J(u) \mid h\rangle+o(t) \geq J(u),
$$

which yields $\langle\nabla J(u) \mid h\rangle=0$.

We now assume that $K=\operatorname{ker} B$, where $B \in \mathcal{L}(V, \Lambda)$, and $\Lambda$ is a Hilbert space, identified to its dual space. We furthermore restrict ourselves to the case of a quadratic functional

$$
v \longmapsto J(v)=\frac{1}{2}|v-U|^{2},
$$

for a given $U \in V$.

Proposition 9 Let $K=\operatorname{ker} B$ be a linear subspace of $V$, and $u$ a local minimizer of $J$ (defined by (47)) over $K$, the linear functional $\xi$ defined in the previous proposition lies in $\overline{B^{\star}(\Lambda)}$.

If we assume that $B$ has a closed range, then $\xi \in B^{\star}(\Lambda)$. If we identify $V$ with its dual space, considering accordingly that $B^{\star}$ maps $\Lambda$ to $V$, it means that there exists $p \in \Lambda$ such that

$$
\left\{\begin{aligned}
u+B^{\star} p & =U \\
B u & =0
\end{aligned}\right.
$$

Conversely, without any assumption on $B$, if $(u, p) \in V \times \Lambda$ verifies (48), then $u$ is the projection of $U$ on $K=\operatorname{ker} B$.

Proof We have $\xi \in K^{\perp}=\overline{B^{\star}(\Lambda)}$ (see e.g. [9]). Now if $B$ has a closed range, $B^{\star}$ has also a closed range, so that $\overline{B^{\star}(\Lambda)}=B^{\star}(\Lambda)$, which yields (48).

Conversely, if (48) is verified, then $U-u \in K^{\perp}$, which implies that $u$ is the projection of $U$ on $K=\operatorname{ker} B$.

Proposition 10 Under the assumption of Proposition 9, if we furthermore assume that $B$ is onto, then $\lambda$ is unique.

Proof This is a straightforward consequence of $\operatorname{ker}\left(B^{\star}\right)=B(V)^{\perp}=\{0\}$. 
Remark 7 Problem (48) is commonly called saddle-point formulation of the constrained minimization problem. Indeed, if we define the Lagrangian of the problem as

$$
L:(v, q) \in V \times \Lambda \longmapsto L(v, q)=J(v)+\langle B v \mid q\rangle,
$$

then $(u, p)$ verifies (48) if and only if it is a saddle point for $L$ in $V \times \Lambda$, i.e.

$$
L(u, q) \leq L(u, p) \leq L(v, p) \quad \forall v \in V, q \in \Lambda .
$$

\section{Unilateral constraints}

We now consider the projection of an element on a closed convex cone $C$. This cone, like all the cones we shall consider in this section, admits the origin as a pole, i.e. $\mathbb{R}_{+} C \subset C$. More precisely, $U \in V$ being given, we aim at minimizing

$$
v \longmapsto J(v)=\frac{1}{2}|v-U|^{2}
$$

over $C$. We denote by $N$ the polar cone to $C$ :

$$
N=C^{\circ}=\{v \in V,\langle v \mid w\rangle \leq 0 \quad \forall w \in C\} .
$$

Proposition 11 (Moreau 1962)

Let $C$ be a closed convex cone in $V$ and $N=C^{\circ}$ its polar cone. Then the identity in $V$ decomposes as the orthogonal sum of the projections on $C$ and $N$. In other words, for any $U \in V$, it holds that

$$
u+\xi=U, u=P_{C} U, \xi=P_{N} U,\langle u \mid \xi\rangle=0 .
$$

Conversely, if $U=u+\xi$ with $u \in C, \xi \in N$, and $\langle u \mid \xi\rangle=0$, then $u=P_{C} U$ and $\xi=P_{N} U$.

Proof For the sake of completeness, we give here a short proof of this standard result established in [25]. Let us first recall that, for any closed convex set, $u$ is the projection of $U$ on $K$ if and only if $u \in K$ and

$$
\langle U-u \mid w-u\rangle \leq 0 \quad \forall w \in K .
$$

Applying this to the closed convex set $C$, and using the fact that $C$ is a cone, we have that

$$
\langle U-u \mid t w-u\rangle \leq 0 \quad \forall w \in C, t \in] 0,+\infty[.
$$

By dividing by $t$, and having $t$ go to $+\infty$, we obtain $\langle U-u \mid w\rangle \leq 0$, i.e. $U-u \in$ $N=C^{\circ}$. Let us now prove that $\xi=U-u$ is the projection of $U$ on $N$. For any $w \in N$

$$
\langle U-(U-u) \mid w-(U-u)\rangle=\langle u \mid w\rangle+\langle U-u \mid 0-u\rangle .
$$




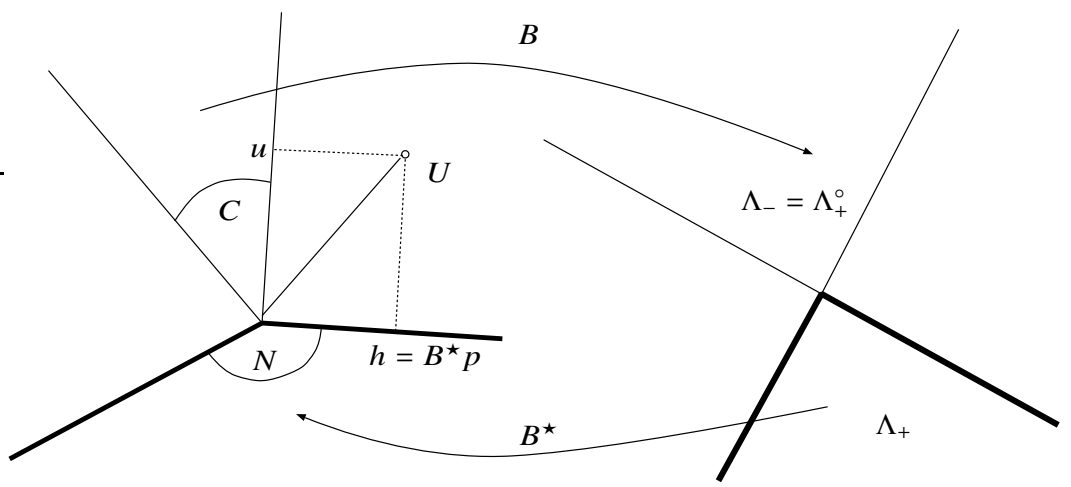

Fig. 11 Mutually polar cones

The first term is nonnegative by polarity, and so is the second one because $u$ is the projection of $U$ on $C$.

Finally, $\langle u \mid \xi\rangle \leq 0$ and, since $0 \in N$,

$$
0 \geq\langle U-\xi \mid 0-\xi\rangle=-\langle u \mid \xi\rangle
$$

so that $\langle u \mid \xi\rangle=0$.

Conversely, if $U=u+\xi$ with $u \in C, \xi \in N,\langle u \mid \xi\rangle=0$, then for any $w \in C$

$$
\langle U-u \mid w-u\rangle=\langle\xi \mid w-u\rangle=\langle\xi \mid w\rangle \leq 0,
$$

so that $u=P_{C} U$. The proof is similar for $\xi=P_{N} U$.

We assume now that $C$ is defined by duality as

$$
C=\left\{v \in V,\langle B v \mid \mu\rangle \leq 0, \forall \mu \in \Lambda_{+}\right\},
$$

where $B \in L(V, \Lambda), \Lambda$ is a Hilbert space identified to its dual, and $\Lambda_{+}$is a closed convex cone in $\Lambda$ (see Figure 11).

Remark 8 One may interrogate the motivation for defining a convex cone by means of another convex cone. This approach will be proven fruitful in many situations where $K$ is natively described in an implicit way, i.e. as the collection of elements which verify certain unilateral constraints, whereas $\Lambda_{+}$is defined in a explicit way, like $\mathbb{R}_{+}^{d}$ in the finite dimensional setting, or as a subset of real functions taking nonnegative values, so that projecting on $\Lambda_{+}$can be computed straightforwardly.

Lemma 2 Let $C$ be a closed convex cone in $V$, defined by (49). It holds that

$$
N=C^{\circ}=\{w \in V,\langle w \mid v\rangle \leq 0, \forall v \in C\}=\overline{B^{\star} \Lambda_{+}} .
$$

Proof For any $\mu \in \Lambda_{+}$, any $v \in C$, it holds $\left\langle B^{\star} \mu \mid v\right\rangle=\langle B v \mid \mu\rangle \leq 0$, so that $B^{\star}\left(\Lambda_{+}\right) \subset N$, so that $\overline{B^{\star}\left(\Lambda_{+}\right)} \subset N$. Now assume that the inclusion is strict: there 
exists $w \in N, w \notin \overline{B^{\star}\left(\Lambda_{+}\right)}$. By Hahn-Banach separation Theorem, there exists $h \in V$, $\alpha \in \mathbb{R}$ such that

$$
\left\langle h \mid B^{\star} \mu\right\rangle \leq \alpha<\langle h \mid w\rangle \quad \forall \mu \in \Lambda_{+} .
$$

Since $\mu$ goes over a cone, the left hand side inequality implies that $\left\langle h \mid B^{\star} \mu\right\rangle \leq 0$ for all $\mu \in \Lambda_{+}$, so that $\alpha \geq 0$ and $h \in C$ by definition of $C$. We then have $\langle h \mid w\rangle>0$, which contradicts the fact that $h \in C, w \in N=C^{o}$.

Let us now introduce the so-called saddle-point formulation of the projection problem

$$
\mid \begin{array}{ll}
u+B^{\star} p=U \\
B u & \in \Lambda_{-} \\
p & \in \Lambda_{+} \\
\langle B u \mid p\rangle & =0 .
\end{array}
$$

Remark 9 The condition $p \in \Lambda_{+}$will correspond in actual applications (impact laws) to $p \geq 0$. It can be written the same way in the abstract setting, if one considers the partial order associated to the closed convex cone $\Lambda_{+}$(see e.g. [12]).

Remark 10 The term saddle-point formulation comes from the fact that there is an equivalence between (50) and $(u, p) \in V \times \Lambda_{+}$being a saddle point for the Lagrangian

$$
L(v, q)=\frac{1}{2}|v-U|^{2}+\langle B v \mid q\rangle,
$$

i.e.

$$
L(u, q) \leq L(u, p) \leq L(v, p) \quad \forall v \in V, q \in \Lambda_{+} .
$$

Proposition 12 Let $C$ be a closed convex cone in $V$, defined by (49), and $U \in V$.

Let $u$ be the projection of $U$ on $C$. If the cone $B^{\star}\left(\Lambda_{+}\right)$is closed, then there exists $p \in \Lambda_{+}$such that $(u, p)$ is a solution to System (50),

Conversely, if there exists $(u, p)$ solution to System (50), then $u$ is the projection of $U$ on $C$.

Proof If $B^{\star}\left(\Lambda_{+}\right)$is closed, it identifies with $N=C^{\circ}$ by Lemma 2, so that there exists $p \in \Lambda_{+}$such that $U=u+B^{\star} p$ by Proposition 11 . Since $U=u+B^{\star} p$ is the decomposition of $U$ over two mutually convex cones (see again Proposition 11), the two terms are orthogonal, i.e. $\left\langle B^{\star} p \mid u\right\rangle=0$

Conversely, if $(u, p)$ solution to System (50), then $u=P_{C} U$ (and $B^{\star} p=P_{N} U$ ), thanks to Proposition 11.

Corollary 1 Under the assumptions of the previous proposition, if $B$ is onto, then Problem (50) admits a solution ( $u, p)$, and it is unique.

Proof Uniqueness is straightforward: if $B$ is onto, then $\operatorname{ker} B^{\star}=B(V)^{\perp}=\{0\}$, i.e. $B^{\star}$ is one-to-one, and there exists at most one $p \in \Lambda_{+}$such that $U=u+B^{\star} p$. Since $B$ 
has a closed range, so does $B^{\star}$ by the Banach-Steinhaus theorem. As a consequence, there exists $\beta>0$ such that $\left|B^{\star} \mu\right| \geq \beta|\mu|$. Now if a sequence $\left(B^{\star} \mu_{n}\right)$, with $\mu_{n} \in \Lambda_{+}$, converges to $w \in V$, then $\left(\mu_{n}\right)$ is a Cauchy sequence by the previous inequality, thus it converges to $\mu \in \Lambda_{+}$, so that $w=B^{\star} \mu \in B^{\star}\left(\Lambda_{+}\right)$.

Remark 11 In the case of a linear space, assuming $B$ has a closed range is enough to ensure that $B^{\star}(\Lambda)$ is closed (see Proposition 9 ). In the case of unilateral constraints, a stronger assumption is needed: $B$ has to be assumed onto for ensuring the closed character of $B^{\star}\left(\Lambda_{+}\right)$. Indeed, the image of a closed convex cone by a linear mapping with closed range is not necessarily closed, even in the finite dimensional setting. Consider e.g. $\Lambda=\mathbb{R}^{3}$, and the parabola

$$
\mathcal{P}=\left\{(x, y, z), z=1, y=x^{2}\right\}
$$

Now consider the closed convex cone spanned by this parabola, i.e.

$$
\Lambda_{+}=\operatorname{conv}\left(\mathbb{R}_{+} \mathcal{P} \bigcup \mathbb{R}_{+} e_{y}\right),
$$

where $e_{y}$ is the unit vector in the direction $y$. The projection of $\Lambda_{+}$on the $(x, y)$ plane is $\mathbb{R} \times] 0,+\infty[\cup\{(0,0)\}$, which is not closed.

Yet, an important family of cones enjoys the property of being linearly mapped onto a closed set, those are the cones spanned by a finite number of vectors.

Lemma 3 Let $V$ be a Hilbert space, and $N$ a convex cone spanned by a finite number of vectors:

$$
N=\left\{\sum_{i=1}^{n} \alpha_{i} G_{i},\left(\alpha_{1}, \ldots, \alpha_{n}\right) \in \mathbb{R}_{+}^{n}\right\} .
$$

Then $N$ is closed, as is its image by any linear mapping.

Proof We give a full proof of this classical result to enlighten the importance of the fact that $N$ is spanned by a finite number of vectors. We prove the result by induction on the number of vectors. For $n=1$, the result is obvious. Assume that the property is true for $n \geq 1$, and consider the cone $N$ associated to $n+1$ vectors. If the $G_{i}^{\prime} s$ are independent, we call $W$ the space spanned by these vectors, and we introduce

$$
G: \alpha \in \mathbb{R}^{n+1} \longmapsto \sum_{i=1}^{n+1} \alpha_{i} G_{i} \in W .
$$

This map is invertible, and its reciprocal $G^{-1}$ is linear and continuous from $W$ to $\mathbb{R}^{n+1}$. Now consider $v^{k}=\sum \alpha^{k} G_{i}$ converging to $v \in W$. then $G^{-1} v^{k}$ converges to $G^{-1} v$, i.e. $\alpha^{k}$ converges toward $\alpha$ in $\mathbb{R}_{+}^{n+1}$ by continuity $\left(G^{-1}\right.$ is a linear mapping between finite dimensional spaces).

Now if the family is linearly dependent, there exists $\mu_{1}, \ldots, \mu_{n+1}$, not all equal to 0 , such that 


$$
\sum_{i=1}^{n+1} \mu_{i} G_{i}=0 .
$$

We consider a sequence $\left(\alpha^{k}\right)$ in $\mathbb{R}_{+}^{n+1}$ such that

$$
\sum_{i=1}^{n+1} \alpha^{k} G_{i} \longrightarrow v
$$

We assume (without loss of generality) that one of the coefficient of (51) is negative. We now consider, for any $k$, the largest $\beta^{k} \geq 0$ such that $\alpha^{k}+\beta^{k} \mu_{i} \geq 0$ for $1 \leq i \leq n+1$. Equality holds for at least one of the indices. Since at least one index $i_{0}$ realizes equality an infinite number of times, we extract the corresponding subsequence (without changing the notation). The limit $v$ writes

$$
v=\lim \sum_{i \neq i_{0}}\left(\alpha_{i}^{k}+\beta^{k} \mu_{i}\right) G_{i}
$$

which lies in the cone spanned by the $n$ vectors $\left(G_{i}\right)_{i \neq i_{0}}$ (by the induction hypothesis), so it is in $N$.

We now address some theoretical issues related to the description of solution sets for the pressure $p \in \Lambda$ for equations of the type (50). Like in the case of equality constraints (Proposition 10), the solution $p$ is unique a soon as $B$ is onto, and uniqueness is lost whenever the range of $B$ is not dense in $\Lambda$. Yet, in the finite dimensional setting, the solution set can be proven to be bounded under some conditions which are typically met for impact laws in granular media. The approach is based on the notion of asymptotic cone (see e.g. [8]):

Definition 2 Let $V$ be a Hibert space, $K \subset V$ a closed convex subset, and $u \in K$. The set

$$
\vec{K}=\bigcap_{t>0} t(K-u)
$$

which does not depend on the choice of $u \in K$, is called the asymptotic cone of $K$ (see e.g. [19]).

Proposition 13 Let $V$ be a Hibert space, and $K \subset V$ a closed convex subset. For any $u \in K$, the asymptotic cone $\vec{K}$ is the set of directions $h$ such that the half line $u+\mathbb{R}_{+} h$ is contained in $K$.

Proof If $u+\mathbb{R}_{+} h \subset K$, then $h$ is in $\vec{K}$ by definition. Conversely, if $h \in \vec{K}, h$ writes $t(v-u)$ for some $t>0$, with $v=u+h / t \in K$, so that $u+\mathbb{R}_{+} h \subset K$.

This notion provides a criterium to identify bounded convex sets (in the finite dimensional setting).

Proposition 14 Let $V$ be a finite dimensional Hibert space, and $K \subset V$ a closed convex subset which contains 0 . Then 


$$
K \text { is bounded } \Longleftrightarrow \vec{K}=\{0\} .
$$

Proof If $K$ is bounded by $M$, then $t K$ is bounded by $t M$, so $\vec{K}$ contains only 0 . Conversely, if $K$ is not bounded, there exists a sequence $\left(u_{n}\right)$ in $K$, with $\left|u_{n}\right| \rightarrow+\infty$. Let $u$ be any element of $K$. Since $V$ is finite-dimensional the unit sphere is compact, and one can extract a subsequence from $\left(u_{n}-u\right) /\left|u_{n}-u\right|$, which converges to some $v \in H$, with $|v|=1$. Now consider $t>0$, and $\theta_{n}=t /\left|u_{n}-u\right|$. By convexity of $K$, it holds that

$$
\left(1-\mu_{n}\right) u+\mu_{n} u_{n}=u+\mu_{n}\left(u_{n}-u\right)=u+t \frac{u_{n}-u}{\left|u_{n}-u\right|} \in K .
$$

Since $K$ is closed, having $n$ go to infinity yields $u+t v \in K$. As a consequence $\vec{K}$ contains the nonzero vector $v$.

Note that the finite dimension is crucial in the previous proposition. Consider for example the case where $V=\ell^{2}$ and $K$ is the hypercube $\left\{x=\left(x_{n}\right) \in V, 0 \leq x_{n} \leq 1\right\}$. The closed convex set $K$ does not contain any half-line, while being not bounded.

We may now establish the main property

Proposition 15 Let $V$ be a finite dimensional Hilbert space, $C \subset V$ a closed convex cone defined by (49), $U \in V$, and $u$ the projection of $U$ on $C$. We assume that $B^{\star}\left(\Lambda_{+}\right)$ is closed, so that (by Proposition 12) there exists $p \in \Lambda_{+}$such that $u+B^{\star} p=U$. If $\operatorname{ker} B^{\star} \cap \Lambda_{+}=\{0\}$, then the solution set

$$
S=\left\{q \in \Lambda_{+}, B^{\star} q=B^{\star} p=U-u\right\}
$$

is bounded.

Proof The solution set can be written

$$
S=\left(p+\operatorname{ker} B^{\star}\right) \cap \Lambda_{+},
$$

it is a closed convex set. Consider $h \in \vec{S}$. By Proposition 13, the half line $p+\mathbb{R}_{+} h$ is contained in $S \subset p+\operatorname{ker} B^{\star}$, which implies $h \in \operatorname{ker} B^{\star}$. Since $S$ is also contained in the cone $\Lambda_{+}$is a cone, it also implies that $p / t+h \in \Lambda_{+}$, for any $t>0$, which yields, by having $t$ go to $0, h \in \Lambda_{+}$. To sum up, $h \in \operatorname{ker} B^{\star} \cap \Lambda_{+}=\{0\}$. We proved that $\vec{S}=\{0\}$, therefore (by Proposition 14), $S$ is bounded.

We end this appendix by defining a notion which is relevant to classify problems according to some sort of abstract maximum principle. In the context of collisions, the issue can be formulated as follows: if the pre-collisional velocity fields tends to violate all the constraints, it can be expected that all contacts will be active, i.e. that all interaction forces will be positive, and the unilateral constraints turn out to be equalities. It is an essential tool to exhibit a Poisson like problem for the pressure in impact laws (see the end of Section 3). We shall see that this intuitive fact is sometimes ruled out, when a general property is not verified. 
Definition 3 (Abstract Maximum Principle)

Let $C$ be a closed convex cone in $V$, associated to $B \in \mathcal{L}(V, \Lambda)$ through Equation (49). Like in proposition 12 , we assume that $B^{\star}(\Lambda)$ and $B^{\star}\left(\Lambda_{+}\right)$are closed, so that, for any $U \in V$, the system (50) admits at least a solution $(u, p) \in V \times \Lambda_{+}$, where $u$ is the projection of $U$ on $C$. We say that the couple $\left(B, \Lambda_{+}\right)$(which encodes the structure of the projection problem) verifies the maximum principle if

$$
B U \in-\Lambda_{-}=-\Lambda_{+}^{\circ} \Longrightarrow \exists p \in \Lambda_{+} \text {s.t. } B B^{\star} p=B U .
$$

Proposition 16 If $B$ verifies the abstract maximum principle defined above then, for any $U$ such that $B U \in-\Lambda_{-}$, there exists a solution $(u, p)$ to $(50)$ such that

$$
B B^{\star} p=B U .
$$

Proof Let us consider the problem with an equality constraint, i.e. $u \in \operatorname{ker} B$. We denote by $u$ the projection of $U$ on $C$. From the maximum principle there exists $p \in \lambda_{+}$such that $B B^{\star} p=B U$, which implies that $u=U-B^{\star} p$ is in $K=\operatorname{ker} B$, so that $u=P_{K} U$ by Proposition 9. Since $B u=0 \in \Lambda_{-}$and $p \in \Lambda_{+}$, the couple $(u, p)$ is also a solution to the problem with unilateral constraints (50), which ends the proof.

Acknowledgements The work of Félicien Bourdin is supported by the ERC grant NORIA, and the work of Bertrand Maury is supported by the ANR project ADHeC.

\section{References}

1. Alder, B. J., Wainwright, T. E. (1959), Studies in Molecular Dynamics. I. General Method, J. Chem. Phys. 31 (2): 459.

2. Ames, W.F., Numerical methods for partial differential equations, Academic press, 2014.

3. Ballard, P., The Dynamics of Discrete Mechanical Systems with Perfect Unilateral Constraints, Arch. Rational Mech. Anal. 154, p.p. 199-274, 2000.

4. Berthelin, F., Existence and weak stability for a pressureless model with unilateral constraint. Mathematical Models and Methods in Applied Sciences, 12 (02):249-272, 2002.

5. Berthelin, F., Theoretical study of a multi-dimensional pressureless model with unilateral constraint, SIAM, Journal on Mathematical Analysis, 2017, vol. 49, no. 3, pp. 2287-2320.

6. Bouchut, F., On zero pressure gas dynamics. Advances in kinetic theory and computing, 22:171-190, 1994.

7. Bouchut, F., Brenier, Y., Cortes, J., Ripoll, J.-F., A Hierarchy of Models for Two-Phase Flows, Journal of Nonlinear Science, 10(6):639-660, December 2000.

8. Bourbaki, N., Espaces Vectoriels Topologiques, Masson, Paris, 1981.

9. Brezis, H., Functional Analysis, Sobolev Spaces and Partial Differential Equations, ed. Springer.

10. Degond, P., Hua, J., Navoret, L., Numerical simulations of the Euler system with congestion constraint, Journal of Computational Physics Volume 230, Issue 22, 10 September 2011, pp. 8057-8088.

11. Degond, P., Minakowski, P., Navoret, L., Zatorska, E., Finite volume approximations of the Euler system with variable congestion, Computers and Fluids (2017), https://doi.org/10.1016/j.compfluid.2017.09.007. 
12. Ekeland, I., Temam, R., Analyse convexe et problèmes variationnels, ed. Dunod.

13. Falk, Richard S., Error Estimates for the Approximation of a Class of Variational Inequalities, Mathematics of Computation, vol. 28, no. 128, 1974, pp. 963-971. JSTOR.

14. Frémond,M., Non-smooth thermomechanics. Springer-Verlag,Berlin, 2002.

15. Golse, F., Saint-Raymond, L., The Navier-Stokes limit of the Boltzmann equation for bounded collision kernels, Invent. math. (2004) 155, pp. 81-161.

16. Joly, P., Some Trace Theorems in Anisotripic Sobolev Spaces, Siam J. Math Anal, Vol. 23, No. 3, pp 799-819, 1994.

17. Liu, C., Zhao, Z., Brogliato, B.: Frictionless multiple impacts in multibody systems: Part I. Theoretical framework. Proceedings of the Royal Society A, Mathematical, Physical and Engineering Sciences, 464(2100), 3193-3211 (2008).

18. Maury, B., A time-stepping scheme for inelastic collisions, Numerische Mathematik, Volume 102, Number 4, pp. 649 - 679, 2006.

19. Maury, B., Analyse fonctionnelle, exercices et problèmes corrigés, Ellipses, Paris, 2004.

20. Maury, B., Preux, A., Pressureless Euler equations with maximal density constraint : a timesplitting scheme, Topological Optimization and Optimal Transport: In the Applied Sciences 17, 333 (2017).

21. Maury, B., Venel, J., A discrete contact model for crowd motion, ESAIM Mathematical Modelling and Numerical Analysis, 45 (2011), 145-168.

22. Maury, B., Roudneff-Chupin, A., Santambrogio, F., A macroscopic Crowd Motion Model of the gradient-flow type, Mathematical Models and Methods in Applied Sciences Vol. 20, No. 10 (2010) 1787-1821.

23. Maury, B., Roudneff-Chupin, A., Santambrogio, F., Venel, J., Handling Congestion in Crowd Motion Modeling, Networks and Heterogeneous Media, Volume 6, Number 3, September 2011, pp. 485-519

24. Maz'ya, V., Sobolev Spaces: with Applications to Elliptic Partial Differential Equations, Grundlehren der mathematischen Wissenschaften, Vol. 342, Springer Science \& Business Media, 2011.

25. Moreau, J.-J., Décomposition orthogonale d'un espace hilbertien selon deux cônes mutuellement polaires, C. R. Acad. Sci. Paris, 255 (1962), 238-240.

26. Moreau,J.J., Some numerical methods in multibody dynamics: application to granular materials, European Journal of Mechanics A/Solids, 13 (4), 93-114 (1994).

27. Nguyen, N.S., Brogliato, B. (2018), Comparisons of Multiple-Impact Laws For Multibody Systems: Moreau's Law, Binary Impacts, and the LZB Approach. In: Leine R., Acary V., Brüls O. (eds) Advanced Topics in Nonsmooth Dynamics. Springer, Cham.

28. Perrin, C., Westdickenberg, M., One-dimensional granular system with memory effects, SIAM Journal of Mathematical Analysis, vol. 50 (6), p.5921-5946 (2018).

29. Ristow G.. Simulating granular flow with molecular dynamics. Journal de Physique I, EDP Sciences, 1992, 2 (5), pp.649-662.

30. Schatzmann, M., A class of Nonlinear Differential Equations of Second Order in Time, Nonlinear Analysis, Theory, Methods \& Applications, 2, pp. 355-373, 1978.

31. Radjaï, F., Dubois, F., (Editors), Discrete-element Modeling of Granular Materials, Wiley 2011.

32. Radjai, F., Roux, S., Moreau, J.-J., Contact forces in a granular packing. Chaos: An Interdisciplinary Journal of Nonlinear Science, AIP, 1999, 9, pp.544-544.

33. Torquato, S., Stillinger, F. H., Jammed Hard-Particle Packings: From Kepler to Bernal and Beyond, Reviews of Modern Physics, 82, July-September 2010.

34. Torquato, S., Truskett, T. M., Debenedetti, P. G., Is random close packing of spheres well defined?, Phys. Rev. Lett., 84 (2000), 2064-2067.

35. Troianiello, G. M., Elliptic Differential Equations and Obstacle Problems, University Series in Mathematics, Springer 1987.

36. Villani, C.: Limites hydrodynamiques de l'équation de Boltzmann [d'après C. Bardos, F. Golse, D. Levermore, P.-L. Lions, N. Masmoudi, N., L. Saint-Raymond]. Séminaire Bourbaki, vol. 2000-2001, Exp. 893. 